\title{
Transient Effects in Lubricated Textured Bearings
}

\author{
Simon Medina ${ }^{a}$, Mark T. Fowell ${ }^{a}$, Sorin-Cristian Vladescu ${ }^{a}$, Tom Reddyhoffa, lan Pegg ${ }^{b}$, \\ Andrew V. Olver ${ }^{\mathrm{a}}$, and Daniele Dini ${ }^{\mathrm{a},{ }^{*}}$ \\ a'Tribology Group, Department of Mechanical Engineering, Imperial College London \\ South Kensington Campus, Exhibition Road, London SW7 2AZ \\ ${ }^{b}$ Ford Motor Company, Dunton, Essex, United Kingdom
}

*Corresponding author: d.dini@imperial.ac.uk

\begin{abstract}
The aim of this paper is to study transient phenomena in hydrodynamic textured bearings. Both convergent and convergent-divergent reciprocating textured bearings are considered. A massconserving formulation of the Reynolds equation recently proposed by some of the authors and used to capture cavitation in steady-state lubricated contacts has been implemented to study transient effects in lubricated textured bearings. It is shown that the proposed solver is capable of capturing the frictional response of bearings characterised by various geometries and loading conditions in both steady-state and transient configurations. Depending on the boundary conditions governing the problems under investigation, changes in load support or film thickness variations are correctly predicted, demonstrating that the methodology developed in this paper is suitable to provide an efficient tool for the design and optimisation of textured bearings.

A qualitative comparison with preliminary experimental data obtained using an apparatus developed to study reciprocating textured surfaces is performed, showing that the characteristic transient behaviour of such surfaces in different loading regimes can be correctly captured using the proposed numerical implementation. Current limitations of the proposed methodology and possible extensions of the technique to study elasto-hydrodynamic and mixed lubrication regimes are also discussed.
\end{abstract}

Keywords: Surface Texture, Lubrication, Transient, Hydrodynamic Bearings, Cavitation, Friction.

\section{Introduction}

Since the pioneering work of Walowit, Allen and co-workers [1, 2], many studies have investigated the potential benefits of adding discrete features to the surfaces of lubricated bearings. A large contribution to this field of study has been made by Etsion and co-workers. In a series of studies they have investigated the effects of texture on friction and lubricant film thickness under differing bearing geometries and operating scenarios [3-6]. An overview of the current status of research into texturing was also produced by the same group [7].

Many theoretical studies of the performance of textured surfaces have utilised the Reynolds equation for thin film flow, discretised using the finite difference approach, along with the Reynolds boundary condition for cavitation onset. However, more recently Ausas et al. [8, 9] have questioned the suitability of non-mass conserving cavitation algorithms of the type based on the Reynolds boundary condition, and used by Etsion and co-workers, for solving textured bearing problems. In these two papers it was clearly shown that load support is over predicted and friction coefficient under-predicted when a non-mass conserving formulation is applied. This criticism has 
been supported by previous contributions of the current authors [10,11], in which a semi analytical mass-conserving formulation of the Reynolds equation including the effects of cavitation was used to show that the critical pressure enhancement in textured bearings occurs downstream of the cavitation reformation boundary and is therefore critically affected by the boundary conditions in the reformation zone. A mass conserving algorithm was identified as key in correctly predicting bearing performance. A number of possible numerical methods are available for solving the cavitation problem while ensuring mass conservation. These have primarily focused on the implementation of the physical model first proposed by Jakobsson and Floberg [12]. This, together with the work by Olsson [13], forms what is widely known as the JFO theory, which utilised non generalised equations in order to correctly locate the cavitation boundary. A fixed pressure value is set in the non-active or cavitated regions while the Reynolds equation is solved within the active or full film counterparts. The principles of this approach were first implemented into a generalised computer algorithm by Elrod and Adams [14] by use of a switch function to determine the approximate location of cavitation boundary during the solution process. This model has been extensively employed in the analysis of lubrication problems and it has been successfully validated by comparison with experimental evidences for both dynamic transient hydrodynamics and elastohydrodynamic lubrication problems [15-17].

The cavitation problem has been widely considered by practitioners interested in free boundary problems for which the mathematical concept of complementarity is a powerful tool. In particular a number of studies have formulated the cavitation problem in terms of variational inequalities [1821], while a parallel group of studies focused on the use of complementarity [22-25]. In these studies the problem of determining the cavitated area was solved using complementarity; however, these methods do not inherently ensure the conservation of mass. This is due to the assumption that the density remains the same throughout the solution domain. This assumption can be reasonably accepted within the active regions; however, in the non-active regions the density varies in both space and time. Giacopini et al. [26] explained how the assumption of constant fluid density throughout the domain leads to the incorrect reformation of the lubricant film. Approaches which merge variational inequalities and JFO theory to produce a mass conserving cavitation algorithm also exist [27]. A common conclusion is that mass continuity is mandatory if an algorithm is to correctly predict the behaviour of thin fluid films in which film rupture and reformation occurs. This is particularly true in cases where cavitation and reformation may occur multiple times within the computational domain of interest (e.g. rough contacts [28], textured surfaces [8, 10, 11, 29-31] and dynamically loaded journal bearings [9, 16]).

The performance of textured bearings has largely been investigated under steady conditions which rarely occur in practice. It is therefore important to consider a number of scenarios in which transient phenomena may well play a part in the performance of textured bearings. In the current paper these will be considered in two specific subsets of cases, firstly in the case of steady sliding in which the sliding surface has a surface texture or pattern, and secondly the case in which a textured surface undergoes transient movement or loading, such as a reciprocating motion. A mass conserving cavitation algorithm based on the mathematical principle of linear complementarity proposed in [26] and extended in [30] is used to study both of these scenarios. Furthermore, an experimental set-up developed to study textured bearing under reciprocating motion will be described and preliminary results will be shown and qualitatively compared with the proposed transient formulation to demonstrate the suitability of the proposed solver to capture the experimental trends. 


\section{Formulation and Numerical Implementation}

\subsection{Complementarity solution of fluid film pressures and cavitation}

The method developed in [26] is used to obtain the lubricant film pressures within the bearing. The method rewrites the isoviscous, compressible Reynolds Equation in a form that presents the two unknown parameters (pressure and density) as two variables ( $p$ and $r$ ) that meet the requirements of a Linear Complementarity Problems (LCPs). This formulation can then be solved using welldeveloped techniques for such problems. This provides a direct solution process that has been found to be very robust and leads to a converged solution within a finite number of computations. The method is also capable of accurately representing pressure distributions with minimal mesh points [26, 30, 32]. It also avoids the use of additional switching functions used by some other methods to distinguish cavitated and full-film lubricant regions. It does so at the expense of requiring a pivoting algorithm to solve the LCP. This can be computationally expensive for a standard single CPU solver; however, this limitation can be easily overcome by making use of multiple-CPUs or graphic processing units (GPUs) implementation, an area in which much research and advance has been made in recent years.

The details of the LCP Reynolds formulation are provided in [26], but the fundamentals and important points are presented here for completeness. As was the case in the group's original contribution [26], the problem is restricted to a one-dimensional thin fluid film (with the associated assumptions, e.g. no side flow), although the technique can equally be applied to two-dimensional surface films [30]. The hydrodynamic analysis is based on the Reynolds equation for compressible fluids in one-dimension:

$$
\frac{\partial}{\partial x}\left(\frac{\rho h^{3}}{6 \mu} \frac{\partial p}{\partial x}\right)-2 \frac{\partial(\rho h)}{\partial t}-U \frac{\partial(\rho h)}{\partial x}=0
$$

This can be used directly if the density, $\rho$, is assumed constant - that is a full film with no cavitation. When both pressure and density can vary within the film, a solution is more difficult. Cavitation is commonly considered as a limiting minimum pressure. The density of oil above this pressure is the standard reference density; cavitation occurs and thus density drops in regions with lubricant pressures at this limit. Some solution techniques divide the film into regions of cavitation and full-film and employ different techniques for each type. The LCP method reformulates the equation so that it can be solved directly using LCP techniques.

A Linear Complementarity Problem is one which can be expressed as:

$$
p=L r+Q
$$

where $L$ is a real matrix, $Q$ is a real vector and $p$ and $r$ are unknown vectors that satisfy the conditions:

$$
\begin{gathered}
p_{i} \geq 0 \\
r_{i} \geq 0 \\
p_{i} r_{i}=0
\end{gathered}
$$


for each component $i$ of the vectors. Various methods have been developed to solve LCP problems and optimised algorithms are possible; using this common formulation allows this technique to take full advantage of computational developments in this field.

Equation (1) does not directly describe a LCP. However, as shown in [26], an LCP version can be obtained. Firstly, the density can be expressed a function of the standard (non-cavitated) pressure:

$$
r=1-\frac{\rho}{\rho_{0}}
$$

and Equation (1) can then, through a number of steps, be rewritten as:

$$
\frac{\partial}{\partial}\left(\frac{h^{3}}{6 \mu} \frac{\partial p}{\partial x}\right)-U \frac{\partial h}{\partial x}-2 \frac{\partial h}{\partial t}+U \frac{\partial(r h)}{\partial x}+2 \frac{\partial(r h)}{\partial t}=0
$$

The discretised form of this equation can then be solved through a finite element [26] or a finite difference [32] approach. The lubricant film is discretised into a number of mesh points at which $p$ and $r$ are to be found. The finite element method is used here to solve the weak formulation of Equation (3) expressed in the form:

$$
[A] p+[B] r+[C]=0
$$

Where $[A],[B]$ and $[C]$ are known matrices. The finite element approach used here is discussed in details, for both one- and two-dimensional problems in our previous contributions [26, 30]. This equation can then be expressed as:

$$
p=-[A]^{-1}[B] r-[A]^{-1}[C]
$$

which matches the required form of an LCP, Equation (2), with $[L]=-[A]^{-1}[B]$ and $[Q]=$ $-[A]^{-1}[C]$. Note that the inverse of Matrix $[A]$ must be obtained prior to solving the LCP; this can be an expensive step for large mesh sizes and so an optimised mesh with variable mesh spacing can lead to significant improvements in computational speed. Alternative algorithms for Horizontal Linear Complementarity Problems (HCLP) could also be used to solve (4) directly (see e.g. [33, 34]) but this has not been attempted as yet.

For a transient solution, all terms in the Reynolds equation must be included. An important point to note is the representation of the term $U \frac{\partial(r h)}{\partial x}$. In order to ensure a positive definite matrix $A$, appropriate test functions must be employed; decentered polynomial functions depending upon the sign of $U$ (direction of motion) can be shown to be the best option for the application under consideration [30].

Once all matrices of equation $\left(p=-[A]^{-1}[B] r-[A]^{-1}[C]\right)$ have been obtained and the LCP matrices $[L]$ and $[Q]$ computed, the LCP solution algorithm is used to calculate the pressure and cavitation $(r)$ variables. The pivoting algorithm described in [26] was used for the results presented here.

\subsection{Dynamic model for bearing at fixed load}

The method described to this point is able to compute the pressures and cavitation state (density) of the lubricant film in a bearing at a point in time. It is a transient solution and therefore relates the current state to the state at the previous step in time. The relevant features of the previous state 
are the density (or cavitation) and the film height at each mesh point ( $r$ and $h$ ). A change in film height at each mesh point can be a consequence of texture moving through the bearing; it can also be a result of separation or approach of the two surfaces as a whole. The latter is referred to as a "squeeze-film" effect since a load-supporting pressure distribution is generated as the two surfaces are squeezed together. Here, we will refer to this as a vertical motion of a surface, taking the sliding motion to occur in the horizontal direction. Thus far, the model described is a transient solution and only relates the lubricant film pressures and cavitation to the texture motion and imposed film thickness. It could be assumed that the overall, or datum, film thickness (neglecting texture features) is held constant. However, this may be unsatisfactory in some cases of practical interest where it is necessary to predict the vertical motion of the bearing in response to the loading. This can be achieved through a dynamic model (rather than purely transient) which considers the vertical motion of the bearing surfaces in response to the applied loading and hydrodynamic pressure.

The dynamic model considered here is a relatively simplified model in which one of the bearing surfaces is fixed in the tangential direction and is not textured. This surface (top surface in the schematics in Figure 1a) is given a finite mass and moves only vertically and at constant attitude angle in response to the varying load support of the lubricant film. The longer, sliding surface (bottom surfaces in the schematics in Figure 1) does not move vertically and the film height is defined relative to this constant datum. A more extensive dynamic model, for example with both surfaces moving, changing attitude angles or loading via springs and dampers, could equally be incorporated but would lead to more variables to consider and possibly disguise the purpose of this work, which is focussed on the effects of the texture on the frictional response of reciprocating bearings.

The bearing is loaded through an applied load on the fixed surface. As a result of the sliding motion, hydrodynamic pressures are generated in the lubricant film producing a load-supporting pressure distribution. These pressures are calculated as described previously. An imbalance between the applied load and oil-film pressure load will cause the upper bearing surface to accelerate up or down. At each time step in the solution algorithm, the current vertical acceleration and velocity of the upper surface are calculated; the position of the upper bearing (i.e. the datum height of the film) for the subsequent time step can then be set as $h_{\text {datum }}=h_{\text {datum, prev }}+v_{\text {vertical }} \times \Delta T$. The new datum film height is used then to calculate the new pressure/cavitation distribution.

This is the simplest form of dynamic model used; it is an explicit solution to the dynamic problem and whilst it is the easiest model to implement and potentially fastest, it can be unstable in certain conditions, particularly for larger time steps. To achieve a more stable solution, an alternative algorithm can be employed in which the datum film thickness (bearing surface vertical movement) is adjusted at each time step in an iterative process until the loads acting upon the bearing are in equilibrium. The squeeze film pressure distribution will depend upon this change in datum film and thus it is (usually) possible to adjust the bearing movement to generate the required pressure load (noting that a separation of the surfaces gives a negative contribution to the load support of the film pressures). This equilibrium of loads can include the inertial load of the upper surface mass or this can be neglected for a massless (kinematic rather than dynamic) solution.

In implementation, the algorithm initially proceeds as for the explicit solution at each time step. Once the film pressures and load support have been calculated, the net load acting upon the upper bearing surface is calculated. If this is within a small tolerance level (a proportion of the applied load) then the solution is stored and the algorithm moves to the next time step. If the net load is 
above the tolerance limit, the datum film is adjusted to act to reduce the load imbalance, and the pressures for the current time step are re-calculated. This procedure is repeated until an acceptably low net load is obtained and the bearing is in instantaneous pseudo-equilibrium. Most of the results presented here use the explicit algorithm; some of the reciprocating results use the alternative, iterative algorithm to improve stability at reversal. The two algorithms used are presented in the flowcharts provided in Figure 2.

Finally, it may be useful to discuss some intricacies of solving such problems in practice. Firstly, the choice of mesh can be critical for transient cases. Whilst it has been found that a relatively course mesh is acceptable for steady-state analyses [26], a finer mesh may be needed for transient analysis. In particular, the mesh must accurately portray the movement of texture features. There are two aspects for this: 1) the texture features must maintain their exact shape between time steps - if they appear to change size due to an inadequate mesh, this appears to provide an additional source or sink for the lubricant. 2) the texture features must not move too far between timesteps - they must slowly move along the fixed surface and not jump from one zone to another. In our analyse, the former is ensured by using a variable mesh with mesh points assigned a small fixed distance from the texture edges, and mesh points assigned to these edges for its position in the current time step and its position in the previous and following time steps to avoid interpolation errors. The time steps must also be small enough to allow accurate convergence of the dynamic solution - the maximum value can depend on the mass for explicit analyses.

Finally, it was sometimes necessary to impose a minimum film thickness for reciprocating analyses; in reality, mixed and boundary lubrication will take over from hydrodynamic load support once the film thickness falls below a critical value - hydrodynamic films of $10 \mathrm{~nm}$ are not realistic. These occurred only for short periods at reversal in some reciprocating bearing analyses.

The main purpose of applying texture to bearing surfaces is generally to reduce friction. For each time step, the friction force is calculated at each mesh point as:

$$
F=\frac{(1-r) U \mu}{h}+\frac{d p}{d x} \frac{h}{2}+p \frac{d h_{L}}{d x}
$$

where $h_{L}$ is the height of the lower surface relative to the bearing datum surface. The net friction force is computed from the integral of these mesh point values. This equation includes a term,

$p \frac{d h_{L}}{d x}$, which is usually neglected in the literature, when often the friction is computed on the untextured flat surface, as proposed in a recent paper by Checo et al. [31].

\section{Results and Discussion}

\subsection{Steady sliding of a convergent textured surface}

To investigate the effect of a moving texture within a bearing and compare the performance against that of both an un-textured and a textured bearing in which the texture is on the stationary surface a simple test scenario of a linear inclined bearing has been developed. The bearing is 5 $\mathrm{mm}$ in length with an outlet film height of $1 \mu \mathrm{m}$, a convergence ratio, $\left(K=1-h_{\mathrm{i}} / h_{0}\right.$ where $h_{\mathrm{i}}$ and $h_{0}$ are the inlet and outlet film thickness values respectively), of $K=0.1$, a lubricant viscosity, $\mu$, of $0.01 \mathrm{Pas}$, and a sliding velocity of $0.5 \mathrm{~m} / \mathrm{s}$. The pocket is $0.5 \mathrm{~mm}$ in breadth and $2 \mu \mathrm{m}$ in height. Both the attitude and film height are fixed throughout the sweep of the pocket through the bearing, and transient terms, apart from those due to the movement of the pocket, are negated through 
selection of this scenario. Global squeeze of the overall bearing (i.e. movement of the two surfaces relative to each other) does not affect the pressure within the lubricant; the key performance indicator of the bearing in terms separation of the surfaces by a lubricant film is therefore the load support (i.e. what load can the bearing support at the current geometry; in reality this would equate to a change in film thickness for the same applied load). Figure 1(b-e) shows the hydrodynamic pressure distribution within the lubricant film at four instances in time as the texture feature (pocket) passes through the bearing. The steady-state pressure distribution within a plain bearing with the same overall geometry and operating conditions is also shown for comparison.

As the pocket begins to enter the bearing, the inlet pressure initially drops due to the local expansion in fluid film thickness; once the entire pocket has entered the bearing (Figure 1 (b)), the pressure at the bearing inlet rises; however, a small region of cavitation remains at the leading edge (outlet side) of the pocket and the pressures in the remainder of the bearing are below those of the plain case. Therefore, the net load support is lower than the equivalent plain bearing at position (b). As the pocket traverses through the bearing the localised transient expansion of the film caused by the pocket (if seen from a frame of reference centred on a column of lubricant) always results in a pressure reduction at the pocket entrance followed by linear rise in pressure (similar to that seen in a step bearing) to a pressure above the plain equivalent. This trend is seen at all pocket positions. When the pocket reaches the position shown in (d), the load support is equal to that of the plain bearing and from that point the load support is greater than that of the plain bearing.

Figure 3 shows the variation in load support and friction force over the duration of the pocket passing through the bearing. The time averaged load support (over 1 complete cycle, pocket traverse) is lower than the plain bearing. The friction force is always lower than the equivalent plain bearing due to the locally increased film thickness reducing the shear stress within the lubricant in the pocket. Variation in friction coefficient largely follows the changes in load support which are significant.

At this point, it is interesting to compare the pressure distribution within a bearing with moving texture against that in a bearing with a stationary texture feature at the same instantaneous location, Figure 4. It can be seen that the two cases have directly opposing behaviour: the stationary case has increased load support relative to a plain bearing with the pocket towards the inlet and decreased load support with the pocket towards the outlet whilst the reverse is true for the sliding texture. This can be anticipated by considering that the direction of oil flow observed from a frame of reference attached to the pocket is reversed between the two cases (i.e. if in each case the pocket is considered as the stationary frame of reference then the opposing walls are sliding in opposite directions).

The scenario in which a single texture feature is a translating pocket is therefore consistent, yet opposite, with the scenario in which the texture feature remains stationary relative to the bearing. It would therefore seem that within the scope of a single translation of the texture feature the bearing experiences conditions at which the addition of texture is both beneficial and detrimental to performance. However, the scenario in which only a single texture feature is contained within the bearing is primarily of academic interest. In most applications it is more likely that a number of texture features will characterise the bearing surfaces. This scenario allows two factors to be investigated, firstly the performance of textured surfaces with multiple textures traversing through the bearing and secondly the interaction between textures. Figure 5 shows the selected pressure distributions as a series of pockets pass through the bearing at different convergence angles. The 
pocket breadth is $0.2 \mathrm{~mm}$ and all other conditions are as for Figure 1, detailed above. At the highest convergence shown in Figure 5(a) $(K=1.0)$, the load support is at all times lower than that of the plain case. At the lowest convergence of $K=0.01$, the load support is at all times above that of the plain case. This agrees with the observations for stationary textures [29], for which a maximum convergence ratio existed above which texture had little or no beneficial effect. Figure 6 shows the effect of pocket spacing on the average load support and average friction force for different convergence ratios. In this case, increasing the density of the pockets (closer spacing) simply intensifies either the beneficial or detrimental effect of texture. These results also follow the general trend observed for stationary texture under steady state conditions [29].

In order to better assess exactly how much the application of texture features to bearing surfaces influences the performance of convergent bearings, the assumption of a fixed film height must be relaxed to mimic the in-service operating conditions of most bearing surfaces. To do this a dynamic model, the global squeeze film and inertias of the surfaces must be taken into account. In all cases shown, the mass of the surface was chosen to be small in order to more easily identify the changes in load generated by the oil film. Figure 7 shows the film thickness and friction force as a series of pockets enters a bearing with conditions given in Table 1. At the start of the motion, the sliding surface is untextured and the minimum film thickness is $1 \mu \mathrm{m}$. As the first pockets enter the bearing, only the inlet is textured, which is detrimental to load support, and the film drops in thickness. The friction, however, also drops since the effect of the greater film thickness of the pocketed regions dominates over the effect of a slightly reduced datum film thickness. Once the textured region passes through the entire bearing, the film thickness begins to rise, eventually reaching a consistent (cyclically varying, steady but not constant) level above the initial level of the plain bearing. Similarly, the friction force drops to a consistent level below that of the plain bearing. Notice that there is continuous variation in both the film thickness and friction force as different pockets enter and leave the bearing (see insets to Figure 7). This variation may be undesirable, leading to vibrations of the bearing, and the extent can depend upon many factors. We do not consider this issue any further here (although this will be the subject of future investigations), and consider only the average film and friction force over a suitable period.

\begin{tabular}{|l|l|}
\hline Bearing Breadth & $5 \mathrm{~mm}$ \\
\hline Pocket size (Width $x$ Depth) & $200 \mu \mathrm{m} \times 2 \mu \mathrm{m}$ \\
\hline Oil Viscosity & $0.01 \mathrm{Pas}$ \\
\hline Speed & $0.5 \mathrm{~m} / \mathrm{s}$ \\
\hline Load & $615 \mathrm{~N} / \mathrm{m}$ \\
\hline Initial Film (Inlet : Outlet) & $1.01 \mu \mathrm{m}: 1.00 \mu \mathrm{m}$ \\
\hline Mass & $1000 \mathrm{~kg} / \mathrm{m}$ \\
\hline
\end{tabular}

Table 1 - Conditions for bearing in Figure 7.

Whilst a full parameter study of the type performed in [29] for stationary pockets is not feasible for transient dynamic cases, many of the observations made previously appear to be true in cases where the texture is moving. Figures 8 and 9 show the effect of variation in pocket spacing, pocket breadth and pocket height - the latter two for the lowest convergence of $K=0.01$ only. Under the conditions of this example, a pocket spacing of $2 \mathrm{~mm}$ is found to optimise the performance of the bearing under consideration. An optimum value might be expected - a very large spacing would clearly be similar to a plain bearing, whilst a very small spacing would have insufficient land area to generate higher pressures (becoming closer to a bearing with an overall thicker film). Interestingly, the effect of the pocket breadth did not show this optimum within the cases considered. Finally, an optimum pocket height of around $1 \mu \mathrm{m}$ was observed - similar to the film thickness. This is in 
accordance with trends observed previously in [29], which showed a strong interdependence between texture and film height in determining the effectiveness of texture.

Finally, it must be emphasised that these observations cannot be over-generalised and are shown only for the example cases considered. There are many interacting factors which determine the effectiveness of texture and the consequences of changing any single design or operating parameter may result in a previously determined optimum parameter becoming detrimental. This was shown previously for bearings with stationary texture [29]. Therefore, it is recommended that a transient analysis of textured bearing surfaces is conducted using efficient tools such as those built on the formulation proposed by the authors at the design stage of newly developed solutions to assess the influence of the texture on the performance of the system.

\subsection{Reciprocating sliding of a convergent-divergent bearing}

In the previous section the more academically interesting case of a plain convergent hydrodynamic bearing was considered under fixed transient (fixed height; moving pockets) and dynamic transient conditions (fixed load; moving pockets). However, many practical lubricated contacts in which surface texturing may be applied undergo reciprocating motion and time dependant loading conditions, an example of which is the piston-cylinder conjunction. In addition, convergentdivergent bearings are more likely to have extended areas of cavitation, and it has previously been observed that the presence of cavitation plays an important role in the effectiveness of texture. Two cases will be considered, firstly the scenario of a plain reciprocating convergent-divergent bearing under constant load and secondly, the same bearing with texture applied to the mid-stroke zone of the bearing. Initially it is important to consider the plain case to establish the baseline behaviour and to validate the model in terms of the behaviours observed. Once the model's qualitative behaviour has been established it will then be used to investigate the effect of adding texture to reciprocating contacts.

\subsubsection{Reciprocating sliding of a plain convergent-divergent surface}

Reciprocating motion leads to a continually varying film thickness as the velocity changes with a sinusoidal profile. Figure 10 shows two distinct behaviours ( $a$ and $b$ with conditions shown in Table 2) that can be observed in the friction values for a plain reciprocating convergent-divergent slider bearing. Case (a) corresponds to a high load scenario in which the film thickness remains low throughout the cycle, high friction is observed at reversals where velocity and film thickness are extremely low (the applied load is high enough to overcome any squeeze film effects at reversal) and drops towards the mid-stroke position as the film thickness increases due to higher entrainment. In case (a) the maximum friction is capped when the film thickness drops below $1 \mathrm{~nm}$ to avoid unrealistically high values. Case (b) represents the behaviour at low loads where the bearing remains lubricated, even at reversal. The friction closely follows the sinusoidal velocity profile but with a spike in friction just after reversal. In Figure 11 the film pressure, film thickness and cavitation zones are shown for various points within a cycle for case. The pressure distributions and more particularly the cavitation profiles near reversal help explain the drop in film thickness and pressure spike just after reversal. As the bearing nears reversal the cavitation at the outlet is fully developed (A). Just after reversal the slider is moving in the opposite direction however the cavity remains, however it is now located in the inlet to the bearing (B). The bearing is effectively starved of lubricant and the film thickness continues to drop despite the entrainment speed increasing. The sliding speed continues to increase however the film thickness does not increase as the remains of the cavity are ingested ( $C$ to $D$ ) into the inlet (the friction reaches a maximum due to increasing shear at a near constant film thickness). Once a stable cavity has 
been reformed at the outlet friction drops and film thickness continues to increase in line with sinusoidal velocity profile. This behaviour is only captured when mass is conserved across areas of cavitation, confirming the importance of the mass conserving formulation.

\begin{tabular}{|l|l|l|}
\hline & Case a) & Case b) \\
\hline Breadth & $2 \mathrm{~mm}$ & $2 \mathrm{~mm}$ \\
\hline Curvature Radius & $40 \mathrm{~mm}$ & $50 \mathrm{~mm}$ \\
\hline Viscosity & 0.05 Pas & 0.01 Pas \\
\hline Motion Amplitude / Freq & $15 \mathrm{~mm} / 2 \mathrm{~Hz}$ & $15 \mathrm{~mm} / 10 \mathrm{~Hz}$ \\
\hline Loading & $2000 \mathrm{~N} / \mathrm{m} \mathrm{(100} \mathrm{kg/m} \mathrm{Mass})$ & $1000 \mathrm{~N} / \mathrm{m}(100 \mathrm{~kg} / \mathrm{m}$ Mass $)$ \\
\hline Mesh Size & $20-40 \mu \mathrm{m}$ & $5-10 \mu \mathrm{m}$ \\
\hline Mesh Size at Pocket Edge & $0.2 \mu \mathrm{m}$ & $0.2 \mu \mathrm{m}$ \\
\hline
\end{tabular}

Table 2 - Conditions for Bearings shown in Figure 10.

In Figure 12, an experimental rig comprising of a flat fused silica plate sliding against a convex AISI 52100 steel pad of width $2 \mathrm{~mm}$ is schematically represented. The pad is $10 \mathrm{~mm}$ long and curved in the sliding direction to give a convergent-divergent form. The silica pad reciprocates relative to the steel with a controlled amplitude and sinusoidal velocity profile via a cam mechanism. Oil is supplied directly to either side of the contact area through two nozzles at a supply rate sufficient to prevent inlet starvation. A load cell is attached to steel pad specimen holder and measures friction force, whilst the film thickness can be measured through the silica pad using either fluorescence or optical-interferometry techniques. Zones of cavitation can also be observed through the silica pad using fluorescence visualisation. The two distinct behaviours noted in Figure $8(a, b)$ can also be observed experimentally, Figure 13. The model and experiment measurements demonstrate consistent behaviour although quantitative agreement is not achieved. This can be attributed to a number of factors both experimental and theoretical, such as the validity of assuming hydrodynamic as opposed to elasto-hydrodynamic lubrication (EHL) in the model and correct alignment in the experimental set up.

\subsubsection{Reciprocating sliding of a textured convergent-divergent surface}

In reciprocating bearings for which the centre and stroke length are known, the positioning and style of texture on the sliding surface can be varied along the sliding extent. For example, the texture could extend along the entire sliding distance or be restricted to the central region and avoid the end-strokes where good boundary lubrication may be important. Figure 14 shows some example cases of reciprocating bearings with and without texture in the central regions only, the pocket spacing, and depth for the five cases considered are given in Table 3. Passage across areas with texture can be identified by the oscillations in friction force and film thickness. As demonstrated by these cases, texture may or may-not give frictional improvements, only one texture results in a film thickness improvement at all points over the plain case, although a number of patterns increase the average film thickness when averaged across the cycle. Friction is reduced by all texture patterns in line with the shear stress reduction within the pocket; overall texture B performs the best. A full parameter study to identify the different cases is not attempted here, but many of the results from the stationary texture study [29] can be applied to these cases. The number of potentially influential parameters for sliding texture is more than those considered for the stationary case and the addition of dynamic factors increases the complexity of any such 
study. However, this model could be applied to practical cases of interest to develop an optimal texture configuration, and could be extended to include more complex dynamic behaviour that would better replicate the real-life conditions.

\begin{tabular}{|c|c|c|c|}
\hline \multicolumn{2}{|l|}{ Viscosity } & \multicolumn{2}{|l|}{0.0252 Pas } \\
\hline \multicolumn{2}{|c|}{ Stroke } & \multicolumn{2}{|l|}{$28.6 \mathrm{~mm}$} \\
\hline \multicolumn{2}{|c|}{ Curvature Radius } & \multicolumn{2}{|l|}{$0.5 \mathrm{~m}$} \\
\hline \multicolumn{2}{|c|}{ Breadth } & \multicolumn{2}{|l|}{$2 \mathrm{~mm}$} \\
\hline \multicolumn{2}{|l|}{ Mass } & \multicolumn{2}{|l|}{$10000 \mathrm{~kg} / \mathrm{m}$} \\
\hline \multicolumn{2}{|l|}{ Frequency } & \multicolumn{2}{|l|}{$4.5 \mathrm{~Hz}$} \\
\hline Texture & Pocket Breadth $(\mu \mathrm{m})$ & Pocket Depth $(\mu \mathrm{m})$ & Pocket Spacing (mm) \\
\hline $\mathrm{A}$ & 20 & 8 & 1.12 \\
\hline B & 20 & 20 & 1.12 \\
\hline $\mathrm{C}$ & 80 & 8 & 0.58 \\
\hline $\mathrm{D}$ & 80 & 8 & 3.38 \\
\hline$E$ & 20 & 20 & 3.32 \\
\hline
\end{tabular}

Table 3-Conditions for bearings in Figure 14.

Finally, Figure 15 shows experimental friction traces with texture applied in the central region of the stroke length. The running conditions of these tests are similar to those presented in Figure 13(a) for smooth surfaces. In this case, the texture has a mildly beneficial effect on friction, which is in qualitative agreement with the predictions shown in Figure 14 obtained for textured surfaces but with different loading and sliding conditions.

\section{Conclusions}

In this paper, the mass-conserving complementarity formulation derived by Giacopini et al. [26] to deal with lubrication problems in the presence of cavitation has been implemented to study the transient behaviour of textured bearings. A number of scenarios have been considered including (i) plain convergent hydrodynamic bearings under fixed transient (fixed height; moving pockets); (ii) plain convergent hydrodynamic bearings under dynamic transient conditions (fixed load; moving pockets); (iii) reciprocating sliding of plain convergent-divergent surfaces; (iv) reciprocating sliding of textured convergent-divergent surfaces.

After discussing the stability of the algorithm and validating the results obtained using the transient solver against steady-state results, specific configurations have been analysed showing the mutual influence that design parameters play in the transient response of textured surfaces. In particular, as already highlighted in [29] for convergent hydrodynamic bearings, the interdependency of many parameters makes optimisation of bearing texture a complex task: not only it is important to consider that poorly chosen texture, or texture operating outside of its design range, can lead to a dramatic decline in performance, but designers should also consider that further worsening can be expected in transient regimes. Another important conclusion is that full optimisation cannot be performed without a mass-conserving formulation and that the use on the unconditionally convergent and stable algorithm proposed by the authors, which also does not require the use of switch functions, seem to provide an ideal tool to study textured bearings.

The study of reciprocating convergent-divergent textured and untextured bearing configurations and the development of both numerical and experimental techniques for their investigations is another novel feature of this paper and the preliminary investigations presented by the authors 
show promise in terms of being able to capture the beneficial (or detrimental) effect that texture may have on the performance of bearings under different operating conditions. This is a significant step to develop our solver into a fully developed design tool.

A comprehensive analysis of different textured surfaces characterised by various loading conditions and stroke lengths and a quantitative comparison between the experimental results obtained using our recently developed in-house test facility and the improved (e.g. incorporating deflections to deal with EHL and three-dimensional textures) transient solver will be the subject of our future investigations.

\section{Acknowledgments}

SM, MTF, AVO and DD acknowledge the support of the Engineering and Physical Sciences Research Council (EPSRC) via grants EP/G026114/1 and EP/K503733/1.

\section{References}

1. Anno, J.N., J. Walowit and C. Allen, Microasperity lubrication. Journal of Lubrication Technology, 1968. 90: p. 351.

2. Hamilton, D.B., J.A. Walowit and C.M. Allen, A Theory of Lubrication by Microirregularities. Journal of Basic Engineering, 1966. 88(1): p. 177-185.

3. Kligerman, Y. and I. Etsion, Analysis of the Hydrodynamic Effects in a Surface Textured Circumferential Gas Seal. Tribology Transactions, 2001. 44(3): p. 472-478.

4. Ronen, A., I. Etsion and Y. Kligerman, Friction-Reducing Surface-Texturing in Reciprocating Automotive Components. Tribology Transactions, 2001. 44(3): p. 359-366.

5. Brizmer, V., Y. Kligerman and I. Etsion, A laser surface textured parallel thrust bearing. Tribology Transactions, 2003. 46(3): p. 397-403.

6. Kligerman, Y., I. Etsion and A. Shinkarenko, Improving tribological performance of piston rings by partial surface texturing. Journal of Tribology, 2005. 127(3): p. 632-638.

7. $\quad$ Etsion, I., State of the art in laser surface texturing. 2005.

8. Ausas, R., P. Ragot, J. Leiva, M. Jai, G. Bayada and G.C. Buscaglia, The Impact of the Cavitation Model in the Analysis of Microtextured Lubricated Journal Bearings. Journal of Tribology, 2007. 129(4): p. 868-875.

9. Ausas, R.F., M. Jai and G.C. Buscaglia, A Mass-Conserving Algorithm for Dynamical Lubrication Problems With Cavitation. Journal of Tribology, 2009. 131(3): p. 031702031702.

10. Fowell, M., I. Pegg, A.V. Olver, A.D. Gosman and H.A. Spikes, Entrainment and Inlet Suction: Two Mechanisms of Hydrodynamic Lubrication in Textured Bearings. Journal of Tribology, 2006. 129(2): p. 336-347.

11. Olver, A.V., M.T. Fowell, H.A. Spikes and I.G. Pegg, 'Inlet suction', a load support mechanism in non-convergent, pocketed, hydrodynamic bearings. Proceedings of the Institution of Mechanical Engineers, Part J: Journal of Engineering Tribology, 2006. 220(2): p. $105-108$.

12. Jakobsson, B.F.L., The finite journal bearing considering vaporization = Das Gleitlager von unendlicher Breite mit Verdampfung. Transactions of Chalmers University of Technology. 1957, Göteborg: Gumpert.

13. Olsson, K.O., Cavitation in Dynamically Loaded Bearings. Transactions of Chalmers University of Technology. 1965, Göteborg: Scandinavian University Books.

14. Elrod, H.G. and M.L. Adams, A Computer Program for Cavitation and Starvation Problems. Proceeding of the First Leeds-Lyon Symposium on Tribology - Cavitation and Related Phenomena in Lubrication, 1974. 
15. Elrod, H.G., A Cavitation Algorithm. Journal of Lubrication Technology-Transactions of the Asme, 1981. 103(3): p. 350-354.

16. Brewe, D., Theoretical modeling of the vapor cavitation in dynamically loaded journal bearings. Journal of Tribology, 1986. 108(4): p. 628-638.

17. Chevalier, F., A. Lubrecht, P. Cann, F. Colin and G. Dalmaz, Film thickness in starved EHL point contacts. Journal of Tribology, 1998. 120(1): p. 126-133.

18. Lewy, H. and G. Stampacchia, On the regularity of the solution of a variational inequality. Communications on Pure and Applied Mathematics, 1969. 22(2): p. 153-188.

19. Laratta, A. and P. Marzulli, Fenomeni di cavitazione in cuscinetti lubrificati: procedimento di calcolo e risultati. Atti del, 1971. 1(0): p. 26-30.

20. Rohde, S.M. and G.T. McAllister, $A$ variational formulation for a class of free boundary problems arising in hydrodynamic lubrication. International Journal of Engineering Science, 1975. 13(9-10): p. 841-850.

21. Cimatti, G., On a problem of the theory of lubrication governed by a variational inequality. Applied Mathematics and Optimization, 1976. 3(2-3): p. 227-242.

22. Kostreva, M.M., Elasto-hydrodynamic lubrication: A non-linear complementarity problem. International Journal for Numerical Methods in Fluids, 1984. 4(4): p. 377-397.

23. Oh, K.P., The numerical solution of dynamically loaded elastohydrodynamic contact as a nonlinear complementary problem. Journal of Lubrication Technology, 1984. 106(1): p. 8895.

24. Oh, K. and P. Goenka, The elastohydrodynamic solution of journal bearings under dynamic loading. Journal of Tribology, 1985. 107(3): p. 389-395.

25. Strozzi, A., Formulation of three lubrication problems in terms of complementarity. Wear, 1985. 104(2): p. 103-119.

26. Giacopini, M., M.T. Fowell, D. Dini and A. Strozzi, A mass-conserving complementarity formulation to study lubricant films in the presence of cavitation. Journal of tribology, 2010. 132(4): p. 041702.

27. Bayada, G. and M. Chambat, Analysis of a free boundary problem in partial lubrication. Quart. Appl. Math, 1982. 40(4): p. 369-375.

28. Almqvist, A., E. Essel, L.-E. Persson and P. Wall, Homogenization of the unstationary incompressible Reynolds equation. Tribology International, 2007. 40(9): p. 1344-1350.

29. Fowell, M.T., S. Medina, A.V. Olver, H.A. Spikes and I.G. Pegg, Parametric study of texturing in convergent bearings. Tribology International, 2012. 52(0): p. 7-16.

30. Bertocchi, L., D. Dini, M. Giacopini, M.T. Fowell and A. Baldini, Fluid film lubrication in the presence of cavitation: a mass-conserving two-dimensional formulation for compressible, piezoviscous and non-Newtonian fluids. Tribology International, 2013. 67(0): p. 61-71.

31. Checo, H.M., R.F. Ausas, M. Jai, J.-P. Cadalen, F. Choukroun and G.C. Buscaglia, Moving textures: Simulation of a ring sliding on a textured liner. Tribology International, 2014. 72(0): p. 131-142.

32. Almqvist, A., J. Fabricius, R. Larsson and P. Wall, A New Approach for Studying Cavitation in Lubrication. Journal of Tribology, 2013. 136(1): p. 011706-011706.

33. Zhang, Y., On the Convergence of a Class of Infeasible Interior-Point Methods for the Horizontal Linear Complementarity Problem. SIAM Journal on Optimization, 1994. 4(1): p. 208-227.

34. Tütüncü, R.H. and M.J. Todd, Reducing horizontal linear complementarity problems. Linear Algebra and its Applications, 1995. 223-224(0): p. 717-729. 


\section{Captions to Figures}

Figure 1 - (a) Schematic of the dynamic bearing; (b-e) pressure distributions generated as a pocket passes through a convergent bearing - the corresponding pressure distribution for a non-textured bearing is shown by the dotted line.

Figure 2 - Flowcharts showing the (a) explicit, and the (b) alternative iterative algorithms.

Figure 3 - Instantaneous load support and friction force during the traversal of a single pocket through a convergent bearing with fixed film thickness. The positions of the pocket at four instances are shown and correspond to the positions and pressure distributions in Figure 1.

Figure 4 - Comparison of the instantaneous pressure distribution generated by a pocket in the sliding surface and the steady-state pressure distribution for a pocket at the corresponding location in the stationary surface (as shown).

Figure 5 - Typical pressure distributions generated by multiple pockets traversing through a bearing at varying convergence ratios.

Figure 6 - An example of the effect of texture spacing on average load support and friction for a convergent bearing with fixed film thickness.

Figure 7 - Transient response of film thickness and friction force as a convergent bearing traverses across a textured surface. The film thickness is initially that of the steady-state condition for a non-textured bearing.

Figure 8 - An example of the effect of texture spacing on average minimum film thickness and friction force for a convergent bearing with fixed applied load.

Figure 9 - An example of the effect of texture breadth and height on average minimum film thickness and friction force for a convergent bearing with fixed applied load. The convergence ratio is 0.01 .

Figure 10 - Two forms of friction force cycles generated by a plain, curved reciprocating bearing under differing operating conditions.

Figure 11 - The pressure and cavitation distribution during the reversal stage of a reciprocating bearing.

Figure 12 - Experimental set-up for measuring the friction and film thickness in a reciprocating bearing.

Figure 13 - Two forms of friction force measured in the reciprocating bearing test rig.

Figure 14 - Friction force and film thickness in a reciprocating bearing with different forms of texture located in the central region only.

Figure 15 - Effect of different texture on friction force, as measured in the reciprocating bearing test rig. 
Figures

a)

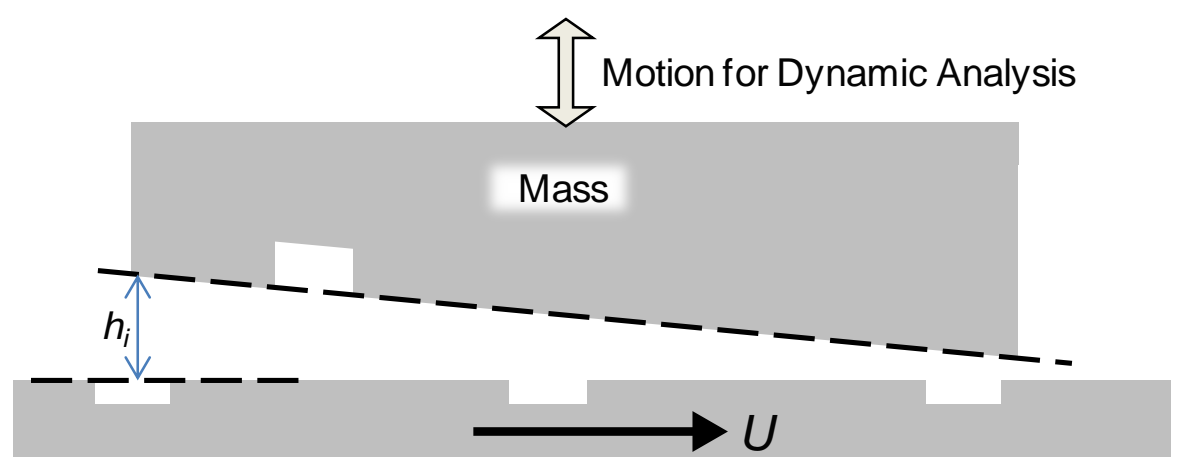

b)

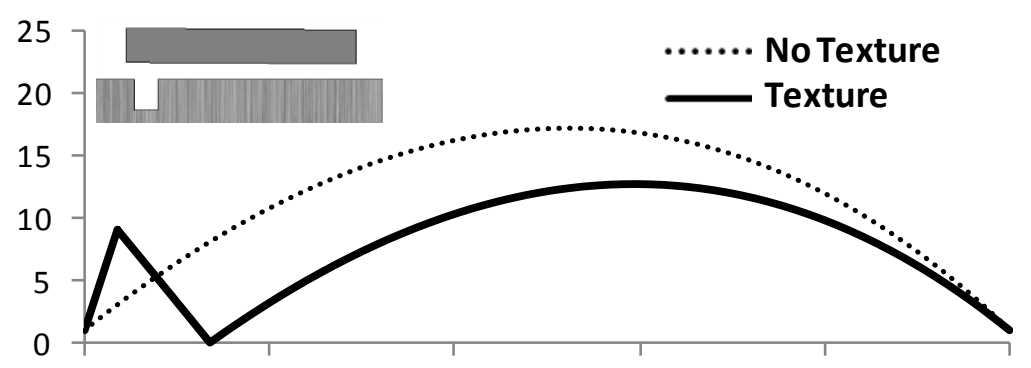

c)

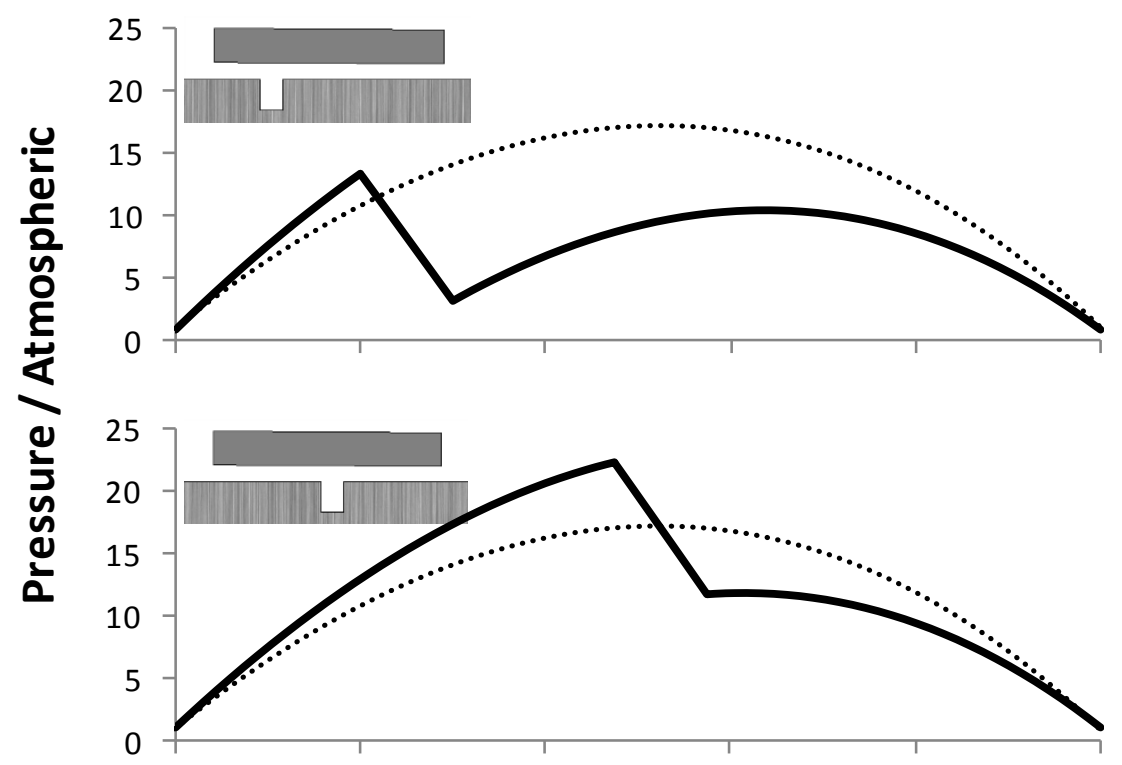

e)

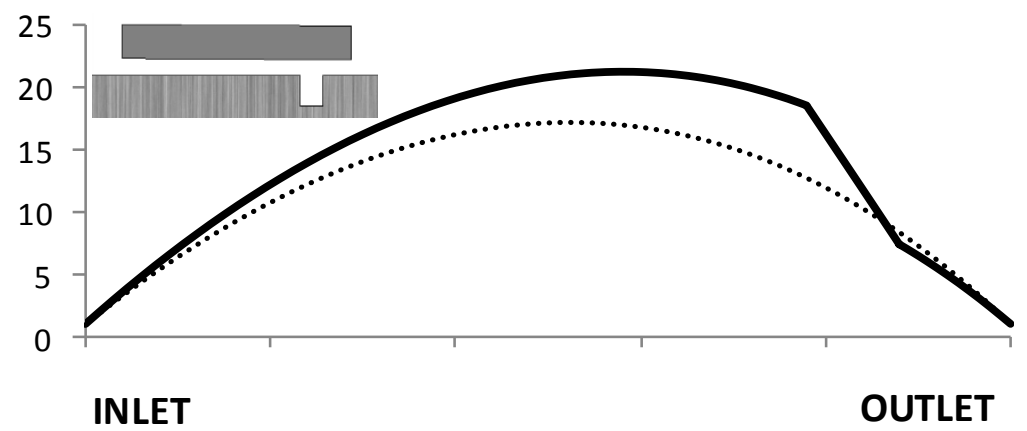

Figure 1 - (a) Schematic of the dynamic bearing; (b-e) pressure distributions generated as a pocket passes through a convergent bearing - the corresponding pressure distribution for a non-textured bearing is shown by the dotted line. 


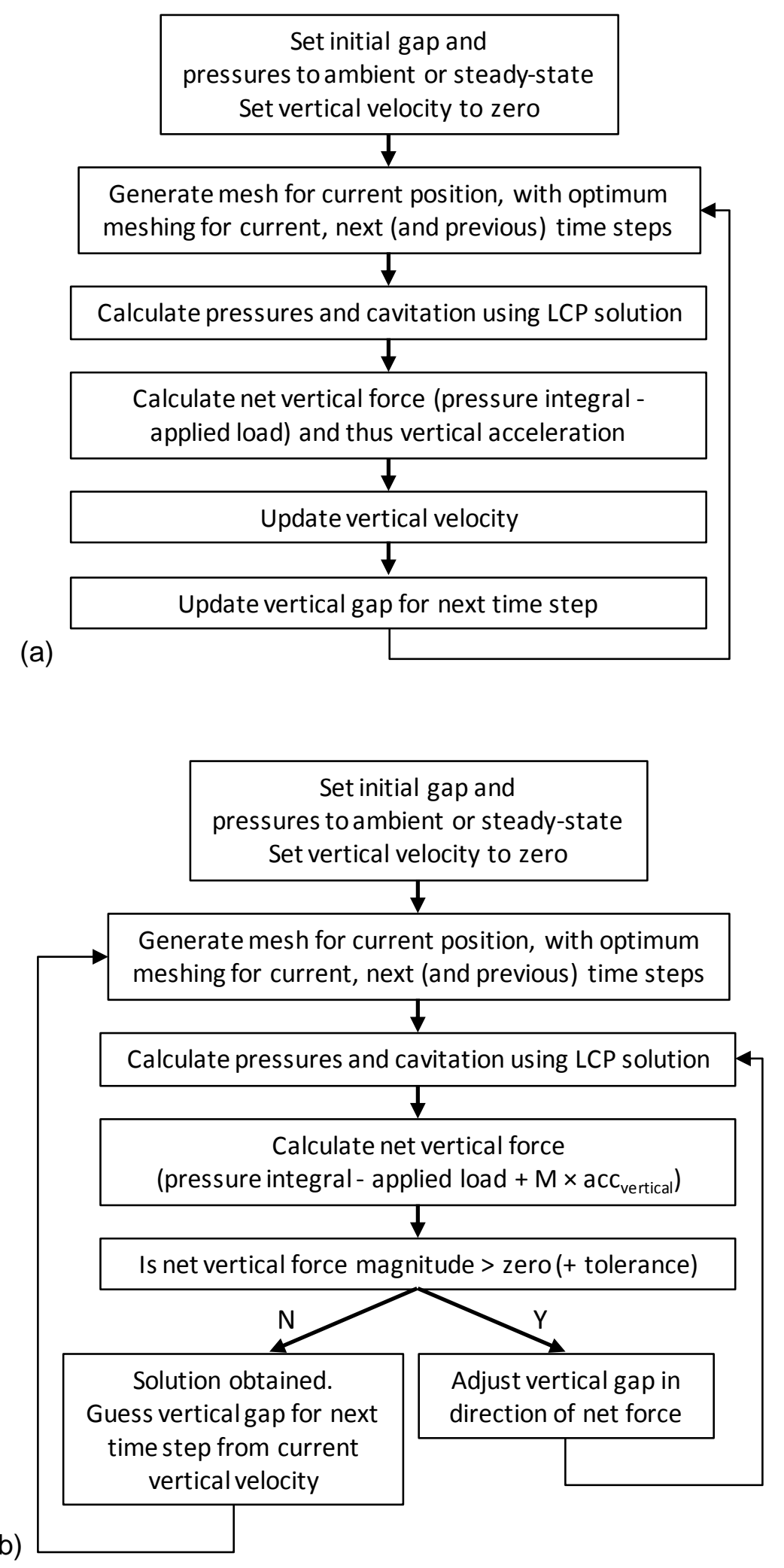

Figure 2 - Flowcharts showing the (a) explicit, and the (b) alternative iterative algorithms. 


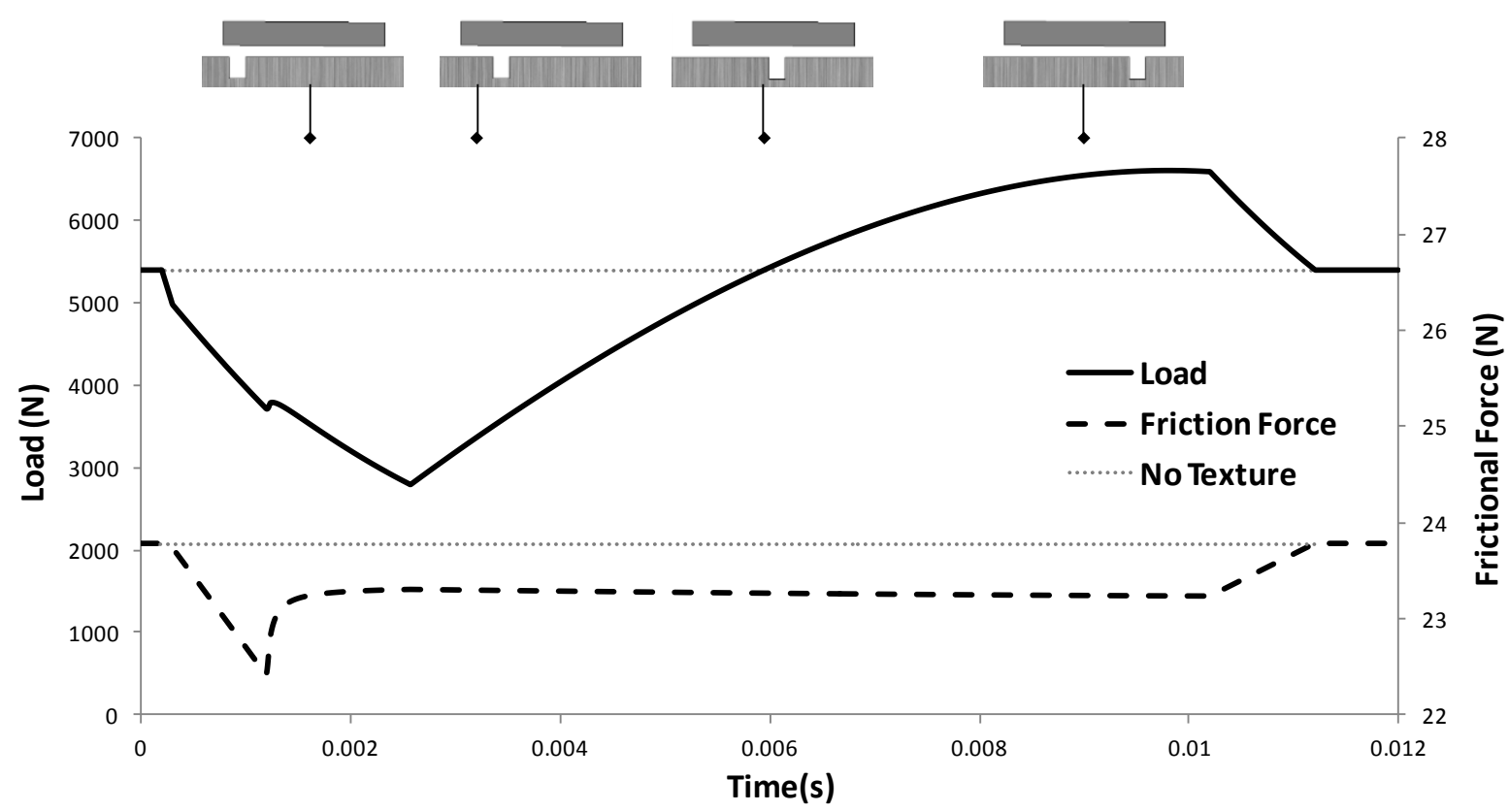

Figure 3-Instantaneous load support and friction force during the traversal of a single pocket through a convergent bearing with fixed film thickness. The positions of the pocket at four instances are shown and correspond to the positions and pressure distributions in Figure 1. 


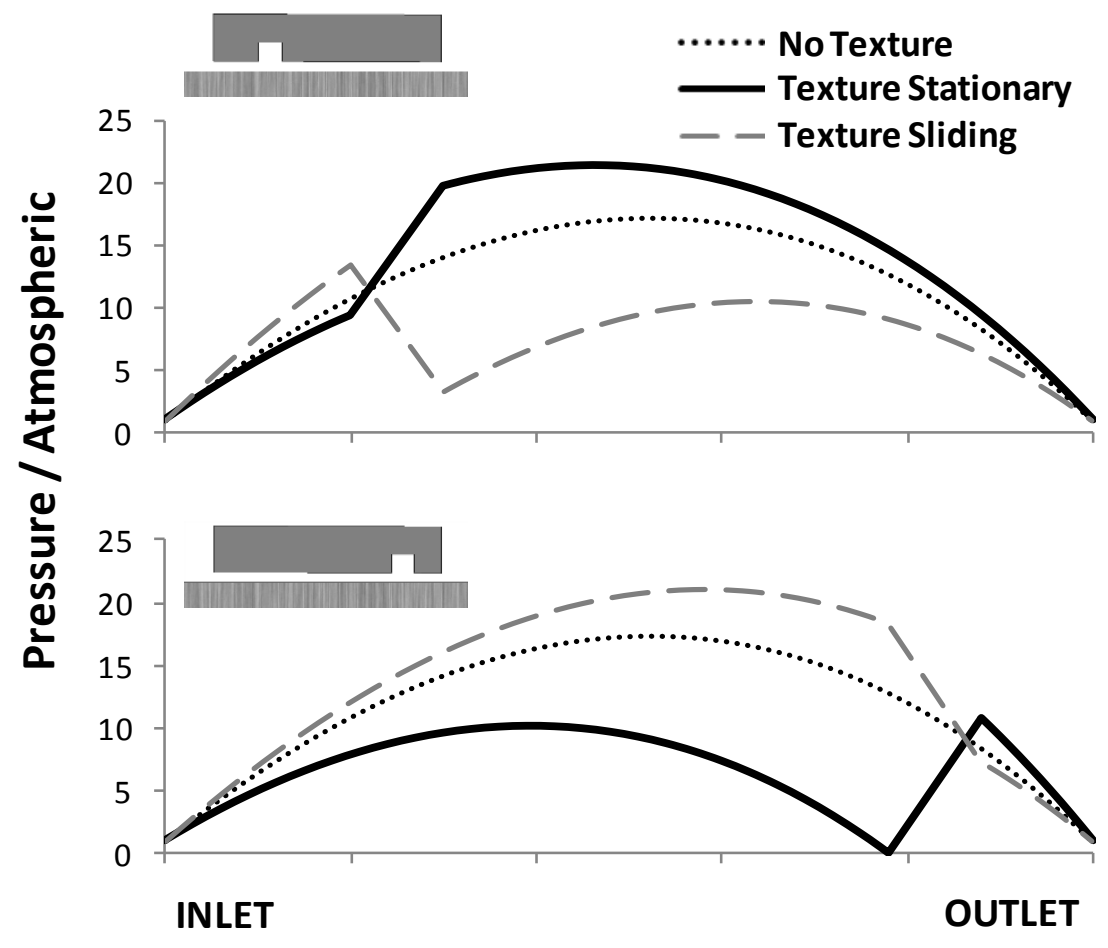

Figure 4-Comparison of the instantaneous pressure distribution generated by a pocket in the sliding surface and the steady-state pressure distribution for a pocket at the corresponding location in the stationary surface (as shown). 

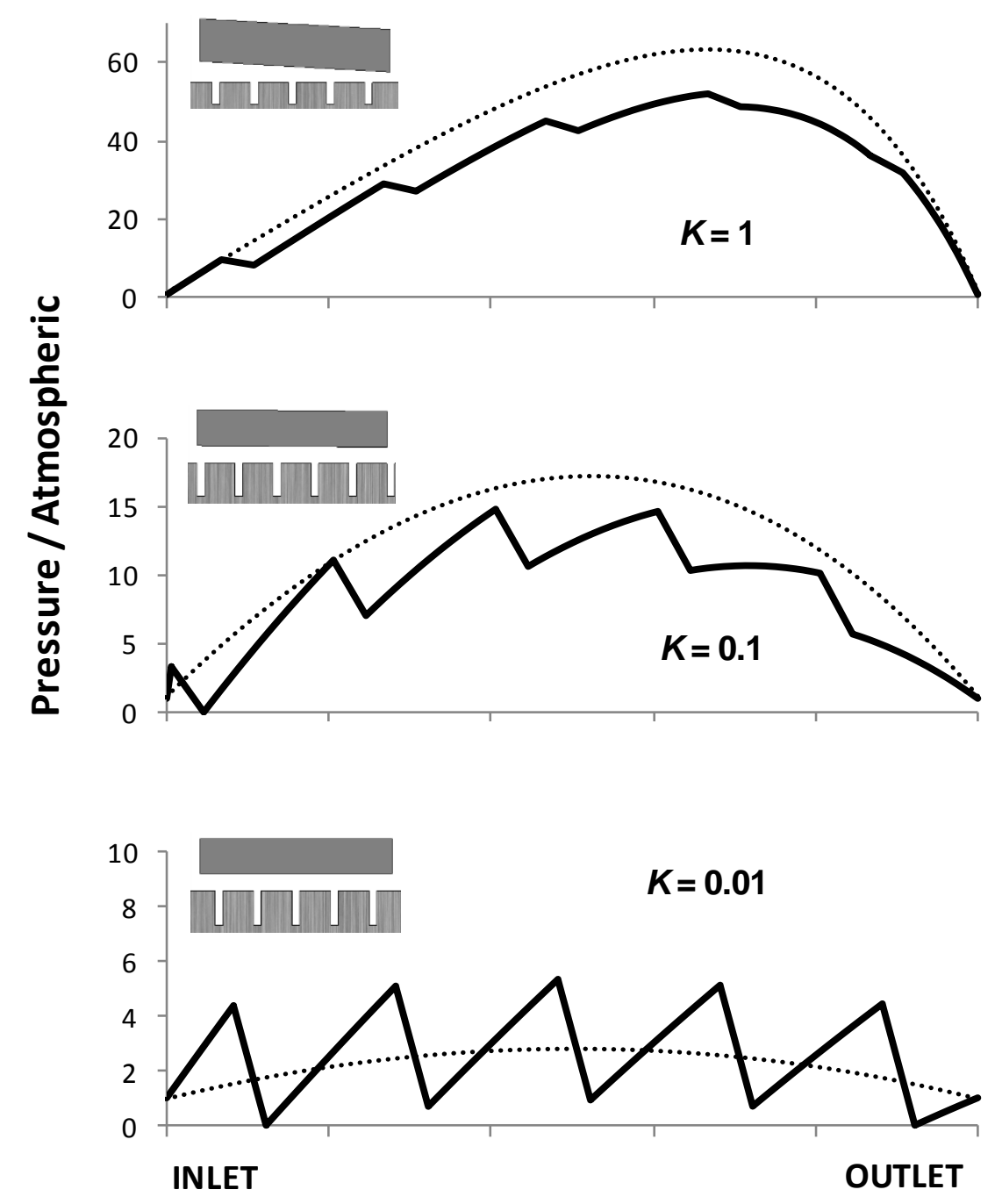

Figure 5 - Typical pressure distributions generated by multiple pockets traversing through a bearing at varying convergence ratios. 


\section{Relative Average Load}

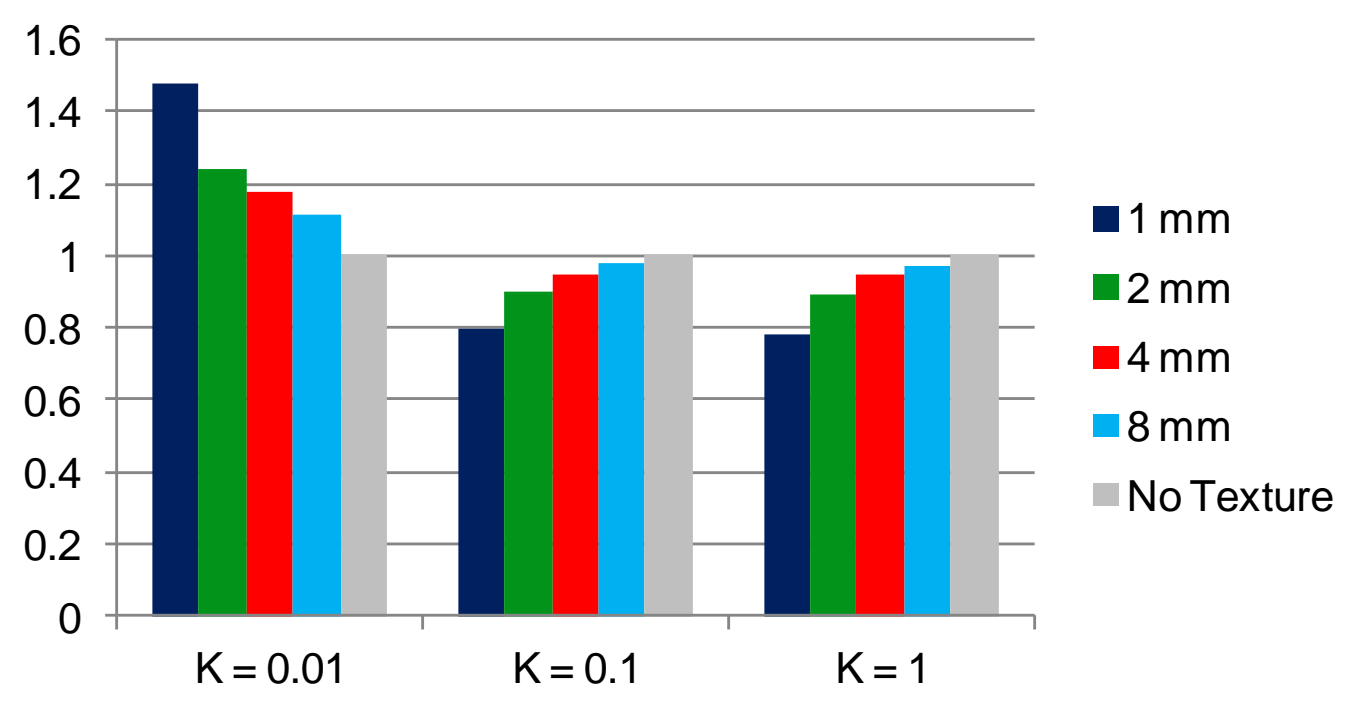

Relative Average Friction

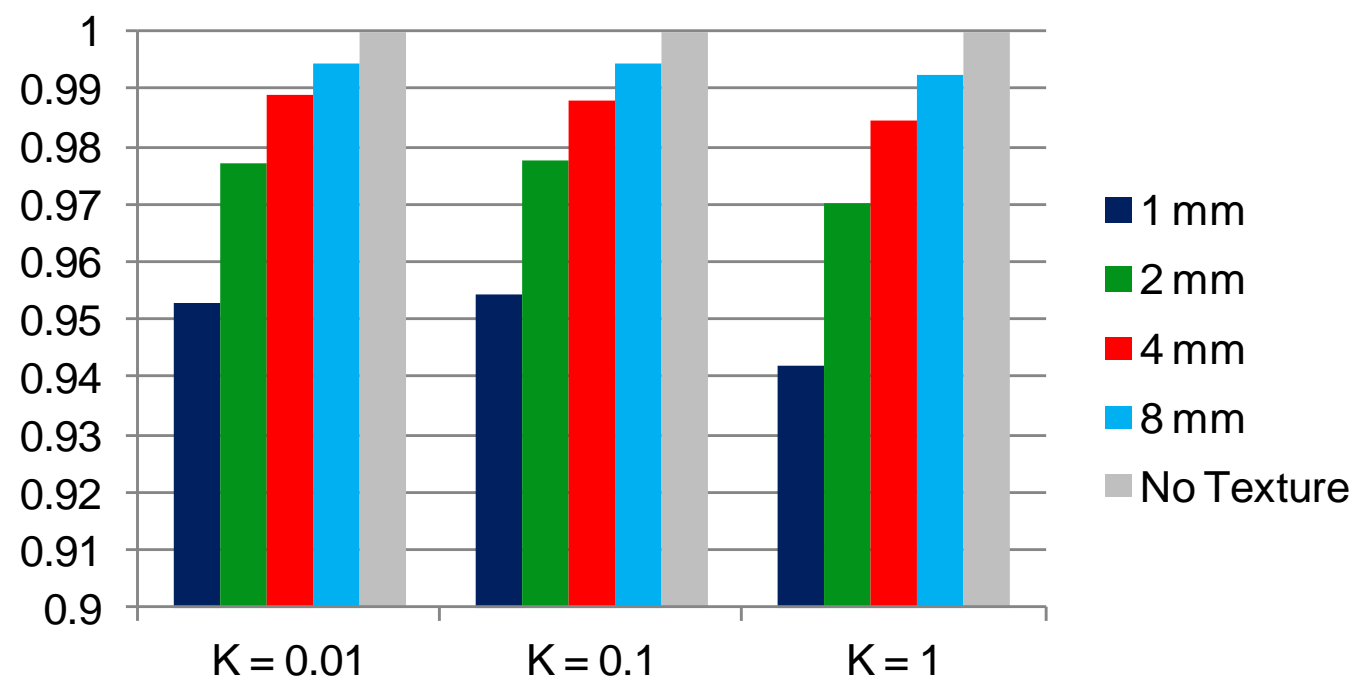

Figure 6-An example of the effect of texture spacing on average load support and friction for a convergent bearing with fixed film thickness. 


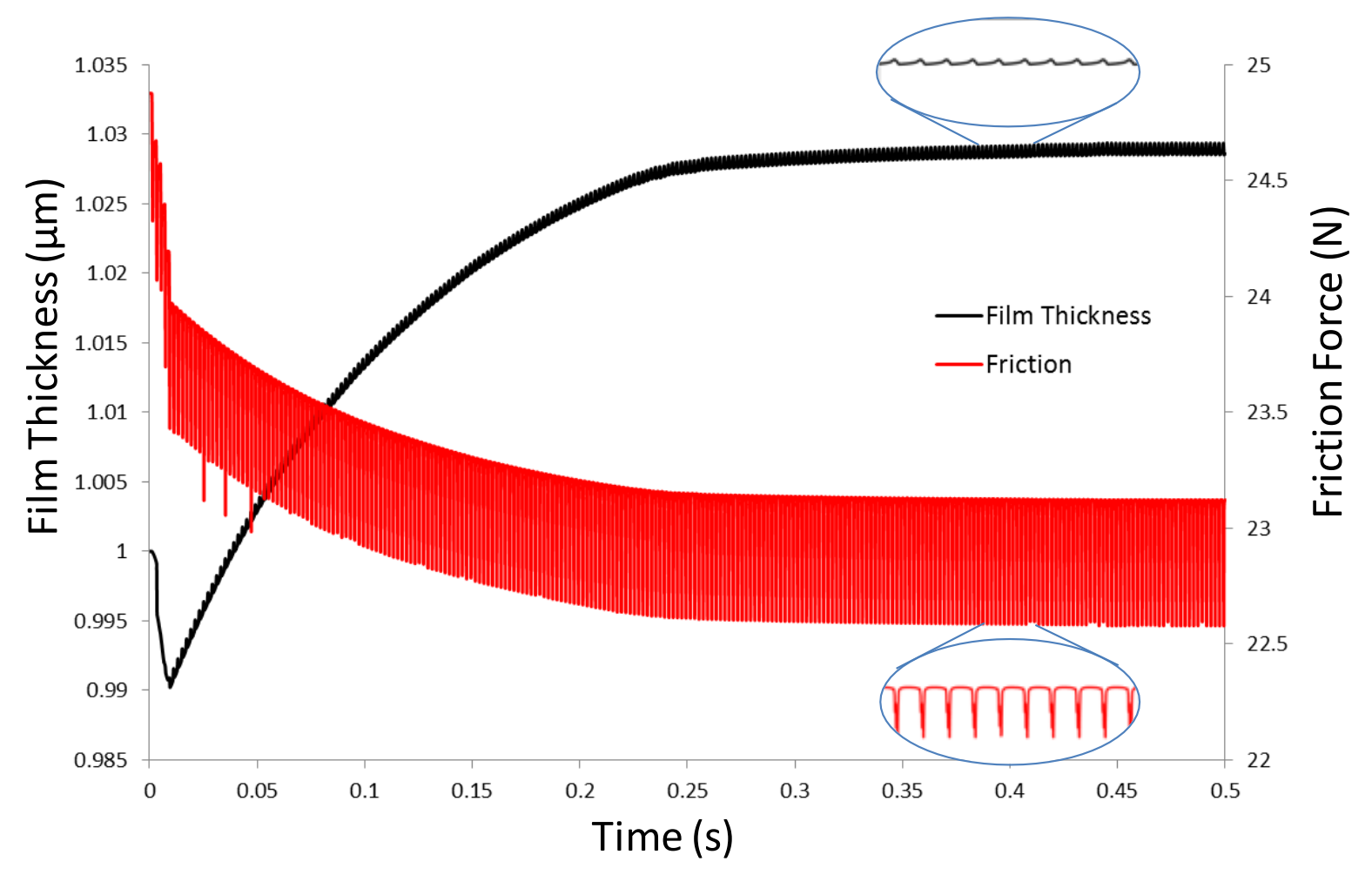

Figure 7- Transient response of film thickness and friction force as a convergent bearing traverses across a textured surface. The film thickness is initially that of the steady-state condition for a non-textured bearing. 


\section{Relative Average Minimum Film}

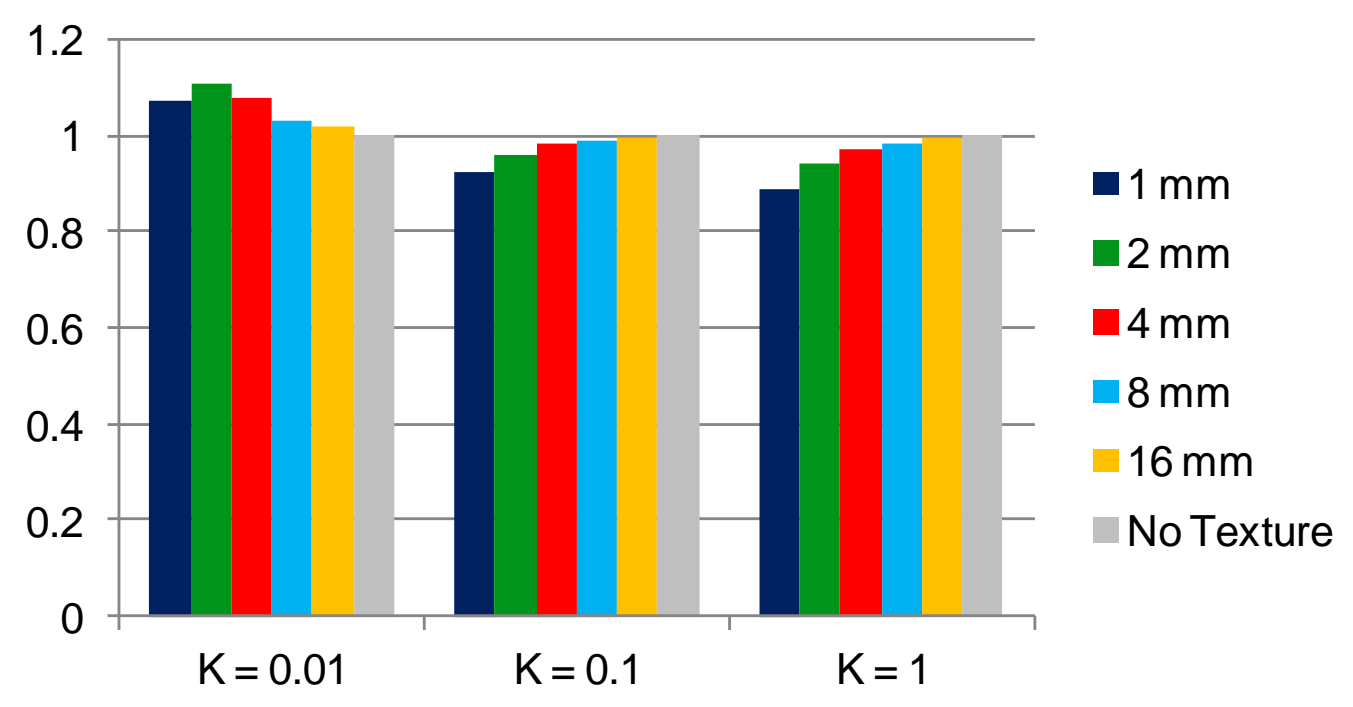

\section{Relative Average Friction Force}

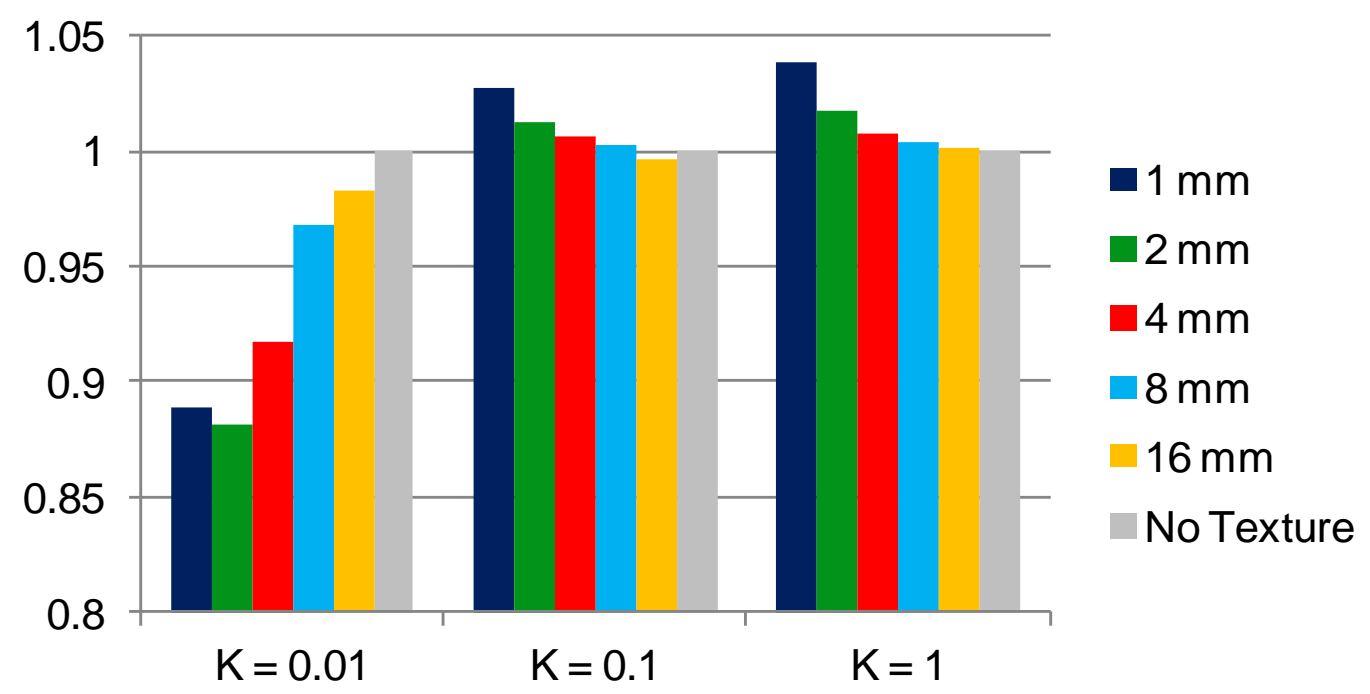

Figure 8-An example of the effect of texture spacing on average minimum film thickness and friction force for a convergent bearing with fixed applied load. 


\section{Effect of Texture Breadth}

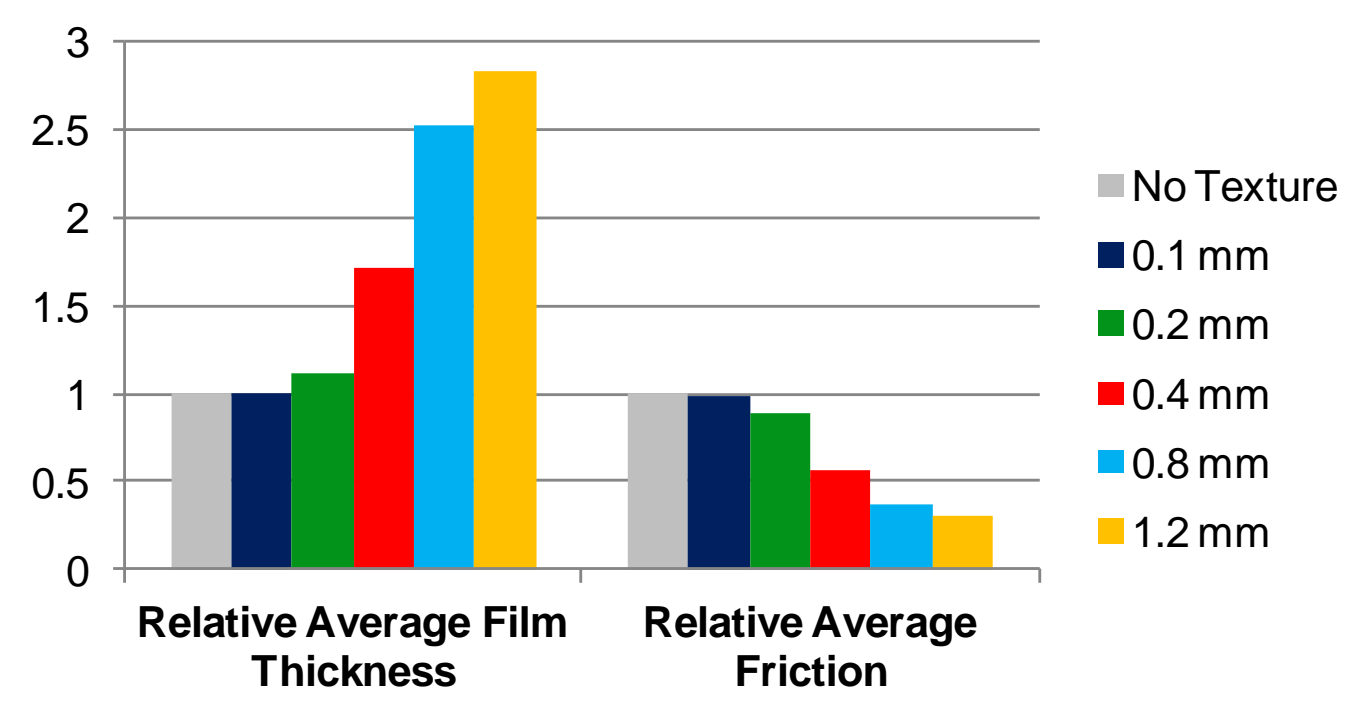

\section{Effect of Texture Height}

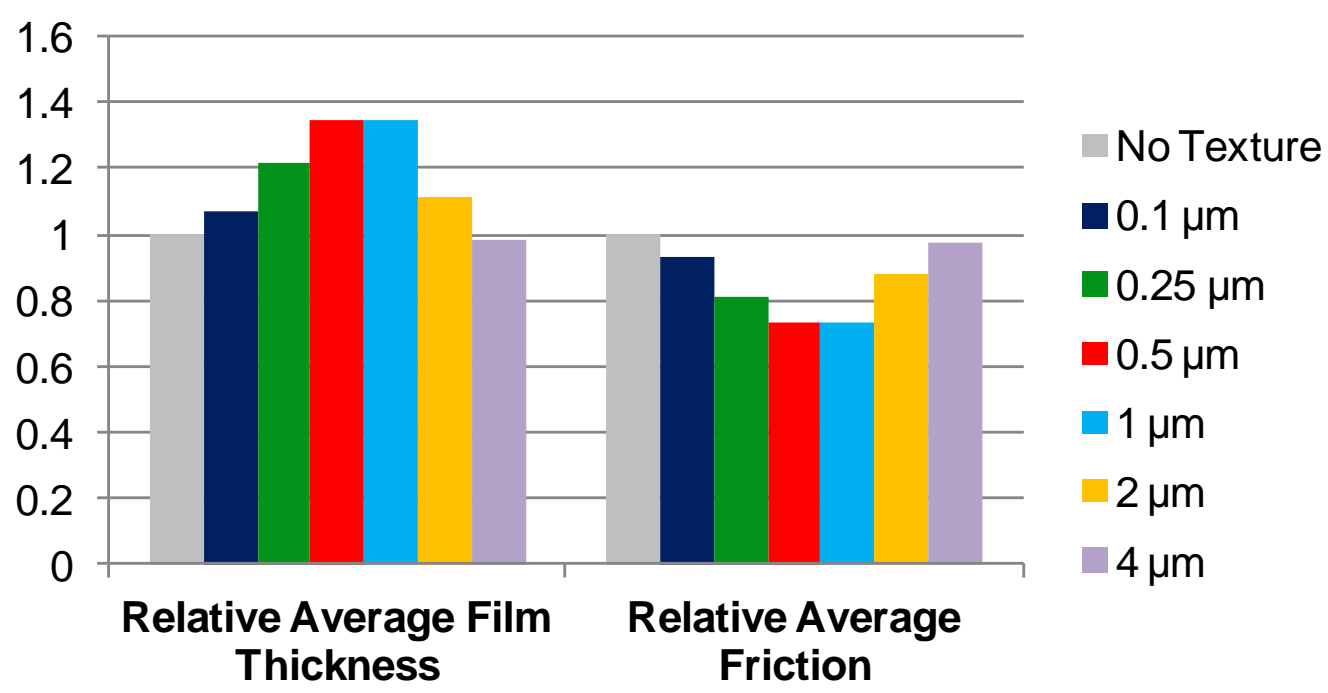

Figure 9-An example of the effect of texture breadth and height on average minimum film thickness and friction force for a convergent bearing with fixed applied load. The convergence ratio is 0.01 . 


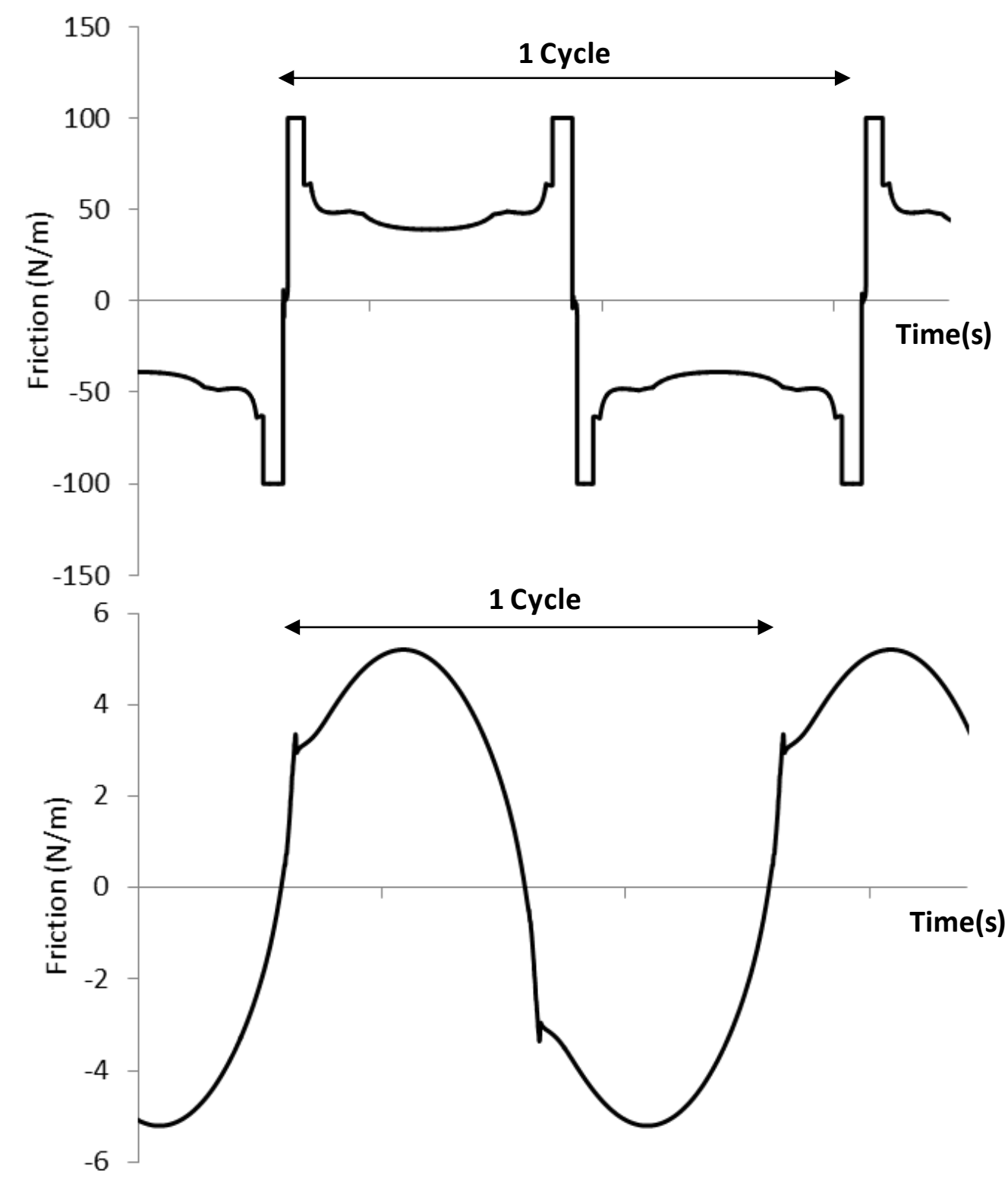

Figure 10 - Two forms of friction force cycles generated by a plain, curved reciprocating bearing under differing operating conditions. 

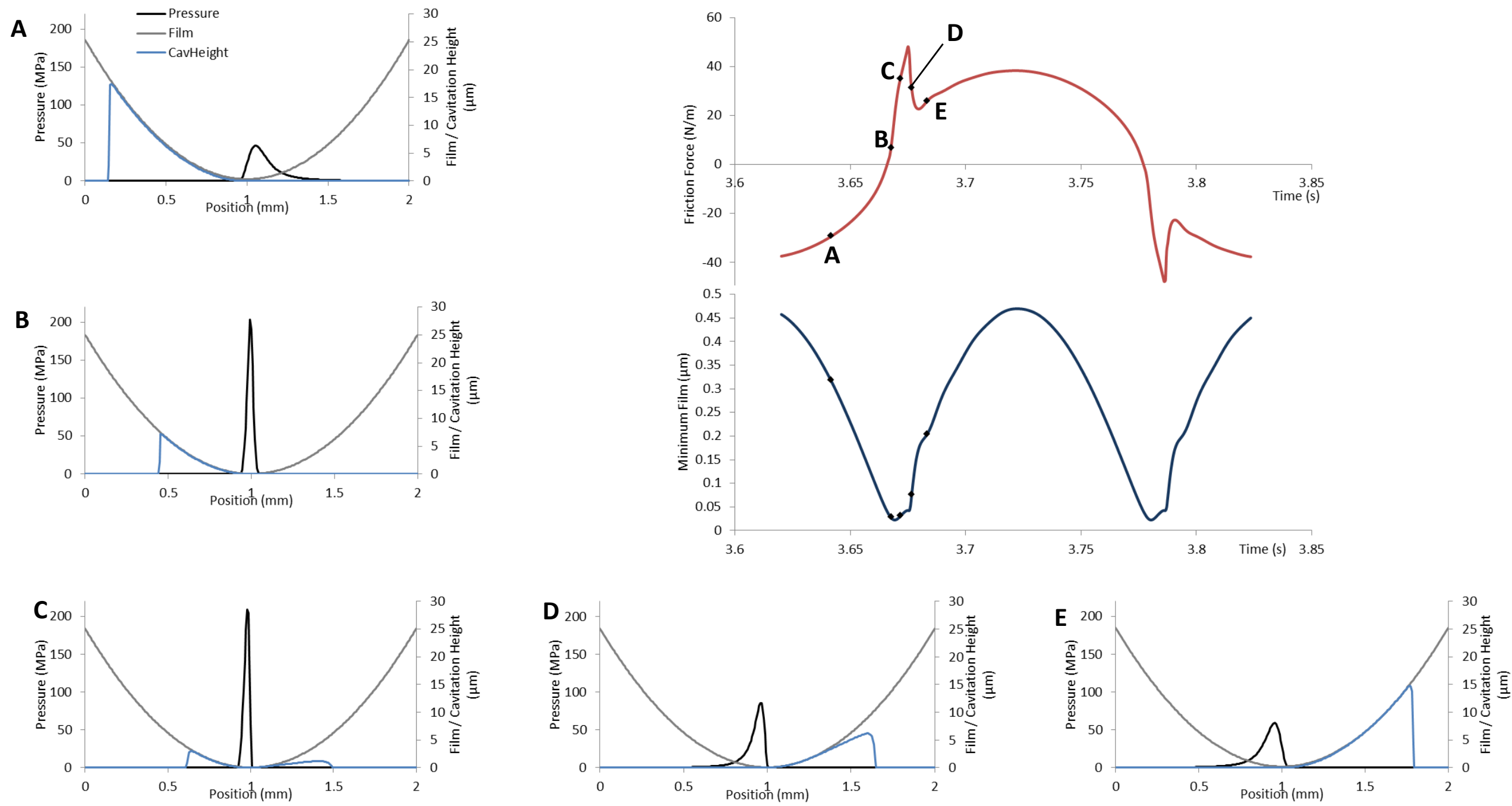

Figure 11 - The pressure and cavitation distribution during the reversal stage of a reciprocating bearing. 


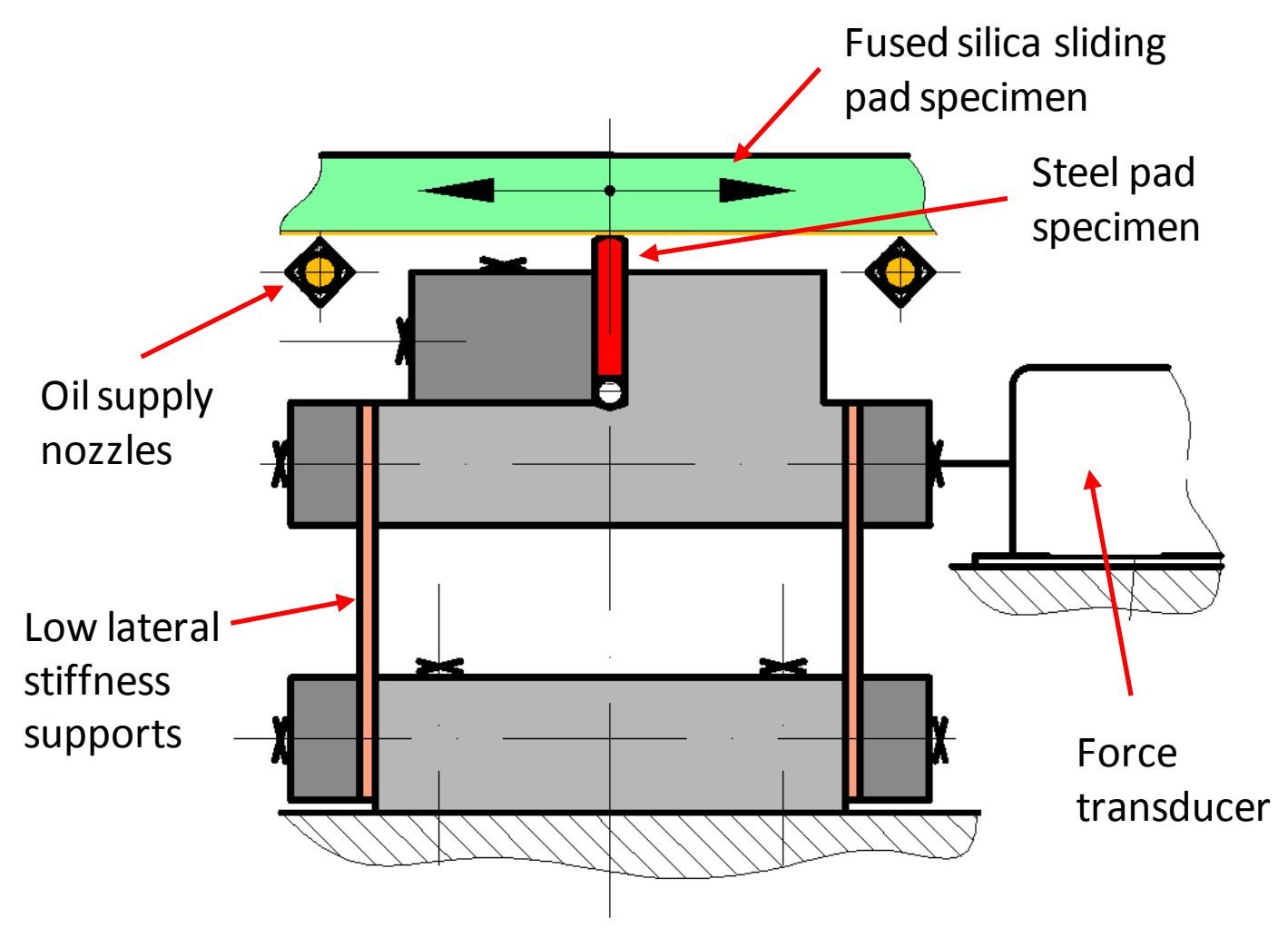

Figure 12 - Experimental set-up for measuring the friction and film thickness in a reciprocating bearing. 

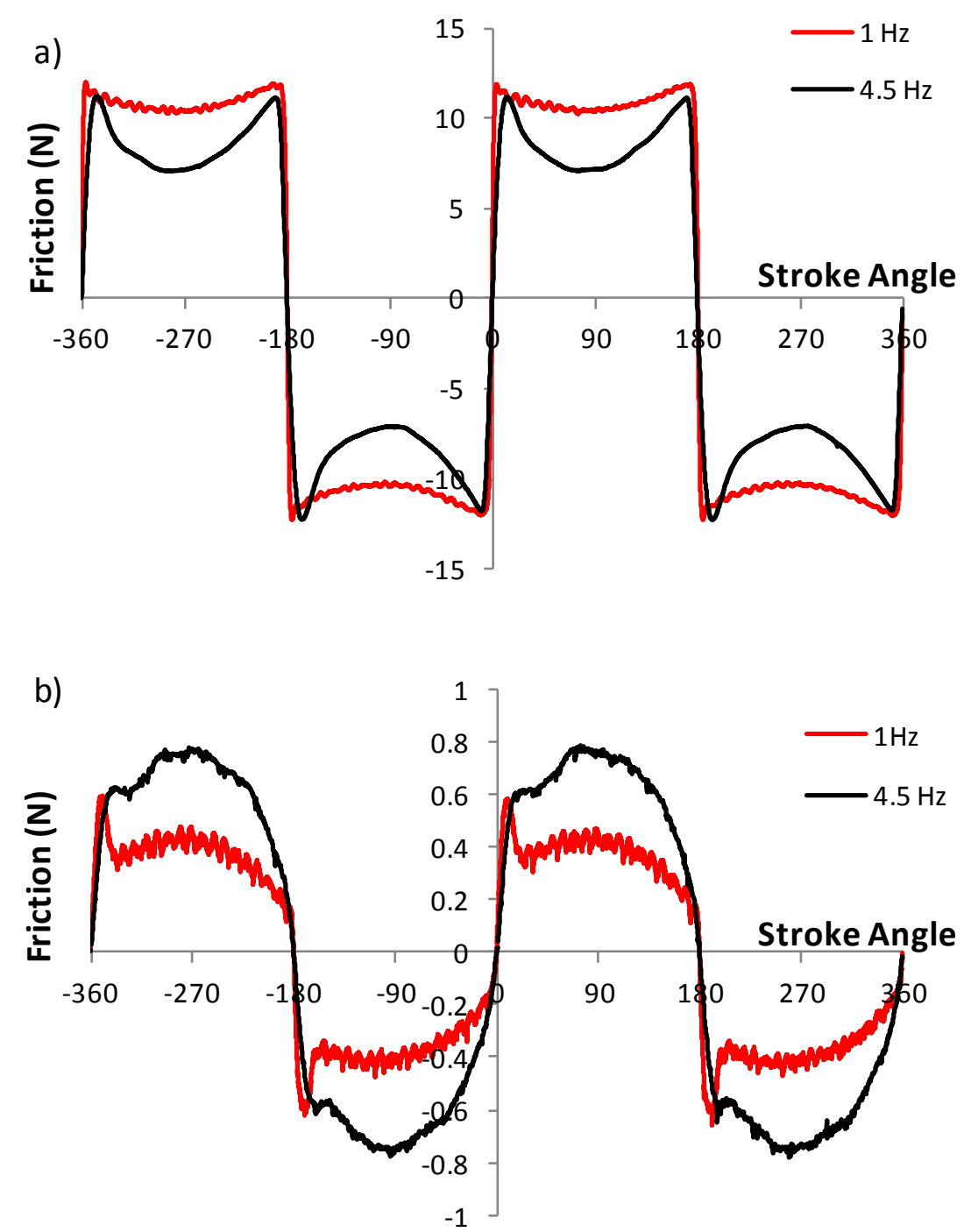

Figure 13-Two forms of friction force measured in the reciprocating bearing test rig. 

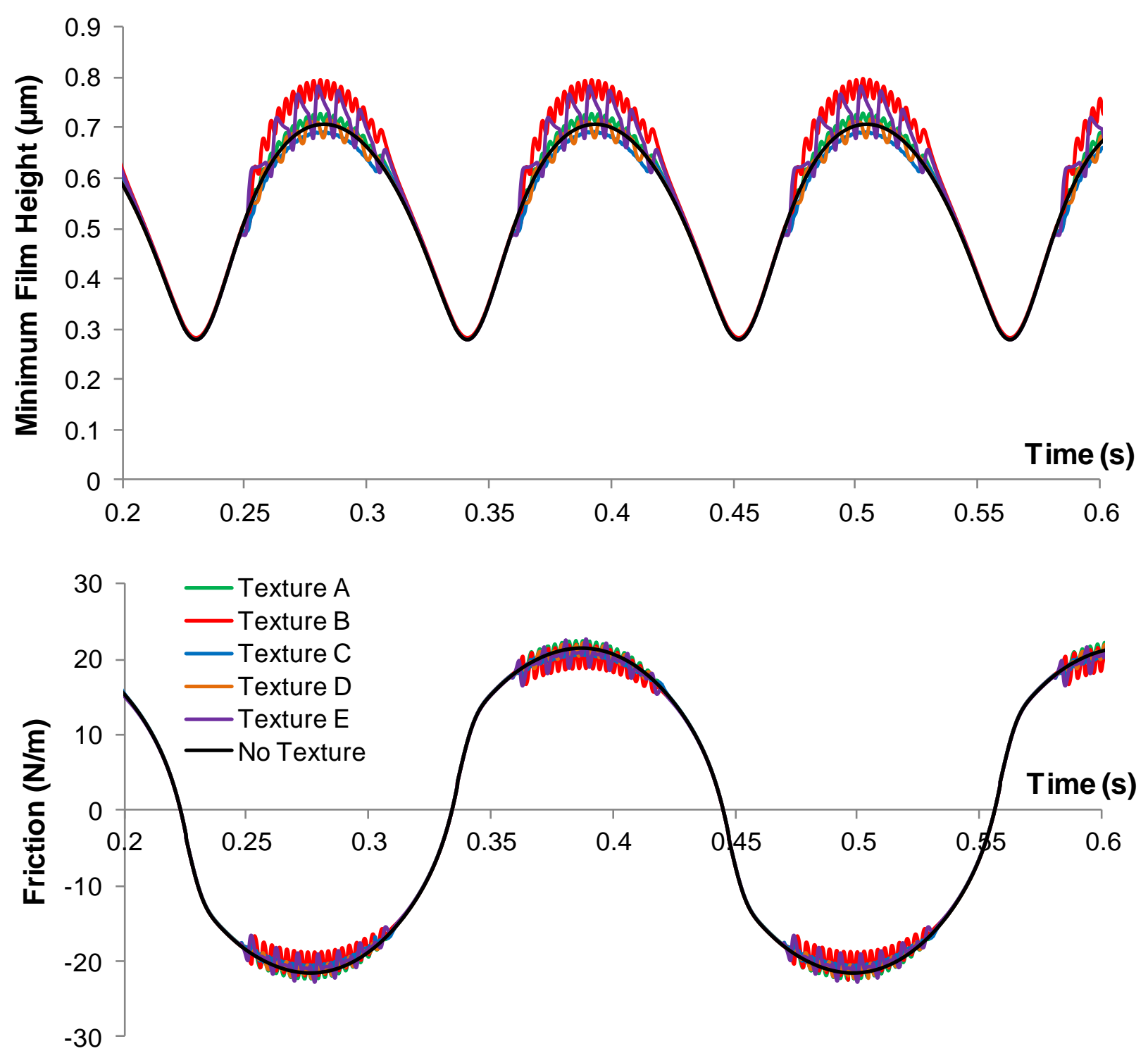

Figure 14 - Friction force and film thickness in a reciprocating bearing with different forms of texture located in the central region only. 


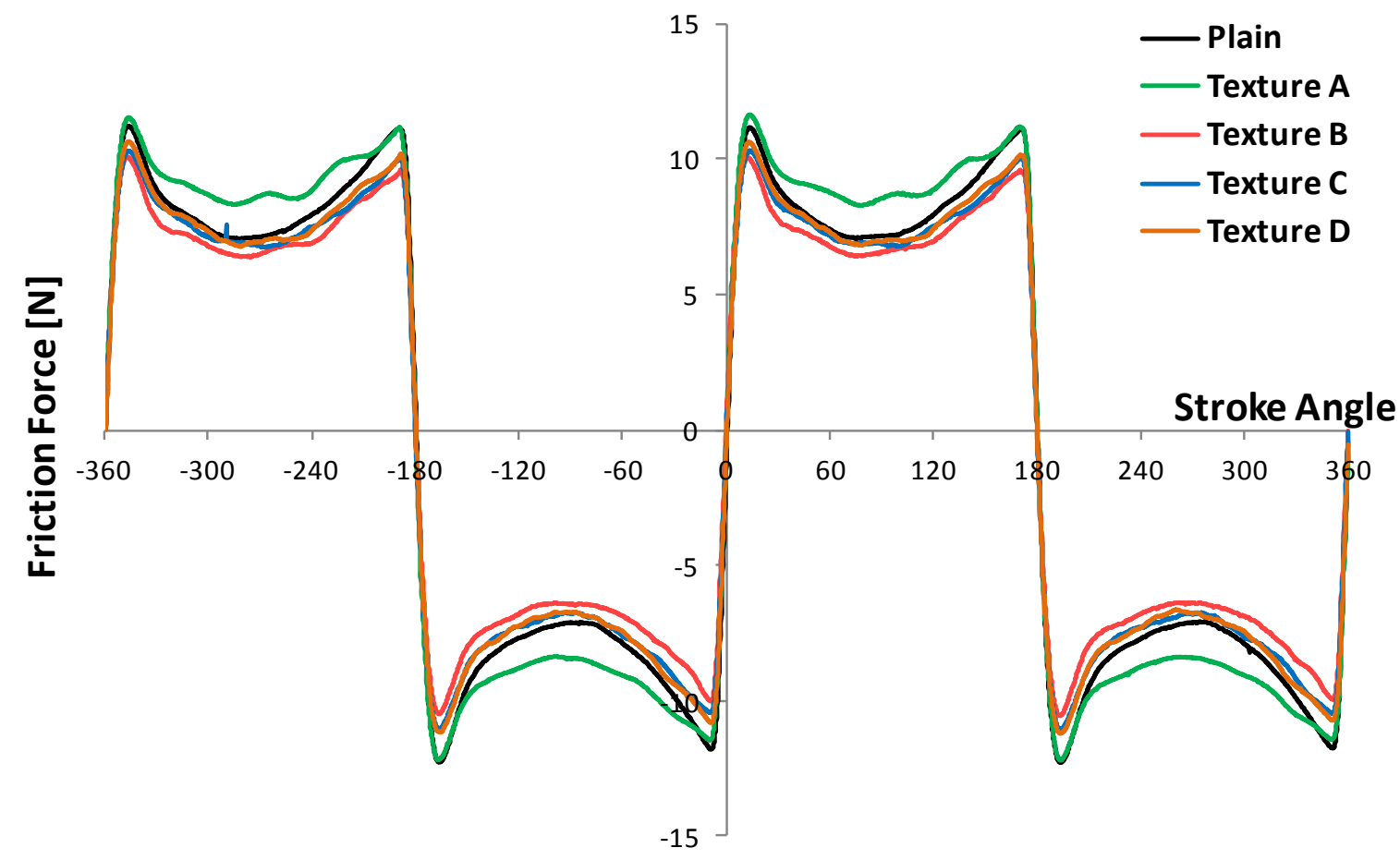

Figure 15 - Effect of different texture on friction force, as measured in the reciprocating bearing test rig. 